$37 \mid 2006$

Langue(s) et religion(s) : une relation complexe dans l'enseignement du français hors de France $x V l^{e}-x x^{e}$ siècle

\title{
Langue(s) et religion(s) en Palestine mandataire au sein d'institutions éducatives catholiques
}

Etablissements des Frères des Ecoles chrétiennes et Sœurs de Saint Joseph de l'Apparition 1922-1940

Karène Sanchez-Summerer

\section{OpenEdition \\ Journals}

\section{Édition électronique}

URL : https://journals.openedition.org/dhfles/72

DOI : $10.4000 /$ dhfles.72

ISSN : 2221-4038

\section{Éditeur}

Société Internationale pour l'Histoire du Français Langue Étrangère ou Seconde

Édition imprimée

Date de publication : 1 décembre 2006

Pagination : $94-133$

ISSN : 0992-7654

Référence électronique

Karène Sanchez-Summerer, «Langue(s) et religion(s) en Palestine mandataire au sein d'institutions éducatives catholiques ", Documents pour l'histoire du français langue étrangère ou seconde [En ligne], 37 | 2006, mis en ligne le 02 juillet 2009, consulté le 27 mai 2021. URL : http:// journals.openedition.org/dhfles/72; DOI : https://doi.org/10.4000/dhfles.72 


\title{
Langue(s) et religion(s) en Palestine mandataire au sein d'institutions éducatives catholiques Etablissements des Frères des Ecoles chrétiennes et Sœurs de Saint Joseph de 1'Apparition $1922-1940$
}

\author{
Karène Sanchez-Summerer \\ Universiteit Leiden et Universiteit Utrecht
}

\begin{abstract}
L'article aborde les relations entre langue(s) et religion(s) en Palestine mandataire, de 1922 à 1940, au sein d'institutions éducatives catholiques. Il s'appuie sur deux études de cas, les établissements des Frères des Ecoles chrétiennes et des Sœurs de Saint Joseph de l'Apparition, qui rassemblent la plus grande partie des élèves francophones. Souvent accusés d'être des missionnaires français trop patriotes par les autorités religieuses, ils proposent une éducation et un enseignement chrétiens sans prosélytisme ouvert à des élèves issus de diverses communautés religieuses. Durant une période qui correspond à la fin du protectorat catholique de la France en Palestine, la langue française est vecteur de religion catholique et la religion catholique vecteur de langue française au sein de ces établissements.

This article deals with the relations between language(s) and religion(s) in British Palestine (1922-1940), within two French catholic institutions, the schools of the Brothers of the Christian schools and of the Sisters of Saint Joseph, which attracted the majority of francophone pupils. Often accused of French patriotism by the religious authorities, they proposed an officially non-proselyte Christian education to pupils from various religious communities. During a period that corresponds to the end of the French Catholic Protectorate in Palestine, the French language is however also used as a vector of catholic religion, while Christian traditions and ceremonies continue to maintain French as a local language.
\end{abstract}


«France Palestine : le trait d'union entre ces deux noms doitil se traduire par le mot église ? » La Croix, $1^{\mathrm{er}}$ mars 1921.

Les établissements tenus par les Frères des Ecoles chrétiennes et par les Sœurs de Saint Joseph ${ }^{129}$ rassemblent la plus grande partie des élèves francophones de la Palestine mandataire (1918-1948). Le pluriel des termes «langue(s) et religion(s)» suggère que d'autres langues et d'autres religions cohabitent avec le français durant cette période tendue de batailles linguistiques et diplomatiques, mettant aux prises les différents acteurs de la langue française avec les institutions religieuses et les religieux qui l'enseignent et la diffusent.

Pionniers de l'éducation francophone, les Sœurs de Saint Joseph et les Frères des Ecoles chrétiennes attirent durant toute la période ottomane les élites chrétiennes essentiellement. Au début du $\mathrm{XX}^{\mathrm{e}}$ siècle, le recrutement s'élargit aux élèves juifs et musulmans ${ }^{130}$. Dans un paysage où «l'inflation scolaire » est importante, les autres collèges d'enseignement secondaire de la Palestine mandataire recrutent en général des élèves de leurs communautés religieuses ${ }^{131}$. Tandis que les Sœurs accueillent une population de filles à grande majorité chrétiennes, la spécificité des Frères, très tôt, est de rassembler tant des élèves chrétiens, à grande majorité des catholiques de rite latin, que des élèves musulmans et juifs. Ces deux ordres, réputés en Palestine

${ }^{129}$ Les différents établissements de Palestine mandataire de ces deux ordres seront pris en compte. Pour les Frères des Ecoles chrétiennes, essentiellement Jérusalem (1878), Jaffa (1882), Caiffa (1884), Bethléem et Nazareth (1893). Pour les Sœurs de Saint Joseph de l'Apparition, premier ordre missionnaire féminin en Palestine au XIX ${ }^{\mathrm{e}}$ siècle, il s'agit des établissements de Jérusalem (1848), Bethléem (1853), Ramleh (1872), Ramallah (1873), Beit Jala (1875), Nazareth (1889), et Naplouse (1904 pour le dispensaire, 1919 pour l'école). Cependant, l'article s'appuie surtout sur ceux de Jérusalem, en raison de la primauté politique, économique et culturelle de la ville, érigée en tant que capitale administrative par les Britanniques, $c f$. NICAUlT, Catherine, Jérusalem 1850-1948, des Ottomans aux Anglais, entre coexistence spirituelle et déchirure politique, Paris : Autrement, 1999.

${ }^{130}$ Le premier collège, celui de Jérusalem, est fondé en 1876, les Sœurs s'établissent à Jérusalem dès 1848 .

131 Il s'agit notamment du collège grec, du collège de Terra Sancta des Franciscains, et du collège du Bishop Gobat, protestant (ouvert dès 1853); cf. PIERRACCINI, Paolo, «Un Universita cattolica a Gerusalemme? L'Opera cardinal Ferrari e il collegio francescano Di Terra Santa », Antonianum (1), 2006. 
mandataire pour le type d'enseignement proposé tant aux familles aisées (collèges) qu'aux familles plus modestes et aux classes moyennes ${ }^{132}$, ne font pas preuve, durant cette période, de prosélytisme en vue de convertir leurs élèves non catholiques ${ }^{133}$. On note que ces liens entre «langue(s) et religion(s)» s'observent dans l'enseignement mais également dans toute l'éducation transmise (celle voulue et celle effectivement transmise), qui dépasse le simple cursus d'enseignement offert par ces différentes écoles. Bien que sous Mandat anglais, ces deux institutions enseignantes continuent à attirer de nombreux élèves ${ }^{134}$.

Ces deux ordres se voient souvent reprocher le fait d'être des missionnaires trop patriotes, à la solde de la France, et en ce sens, ils sont accusés de ne pas être les acteurs d'une transmission souhaitable de la religion par les autorités religieuses centrales (Patriarcat latin, Propaganda Fide, et par la même, le Vatican). Ces rapports touchent donc les acteurs au sein même des collèges et des écoles, la plupart des Frères et des Sœurs étant de nationalité française ${ }^{135}$.

La langue française, telle qu'elle apparaît dans les politiques linguistiques britanniques et anglaises, est une langue tantôt voulue tantôt rejetée (politiques de promotion, de diffusion, de lutte). Il s'agit d'une langue «instrumentalisée » par les autorités mandataires, consulaires françaises et religieuses (locales et romaines), puisqu'on

\footnotetext{
${ }^{132}$ Généralement un établissement comporte une section payante pour les classes plus aisées et une section gratuite. Les archives, notamment les correspondances avec la Maison Généralice et avec le Consulat Général, font état du manque de place, de l'exiguité des locaux qui doivent accueillir les 2 sections.

${ }^{133} \mathrm{Il}$ y a pourtant une volonté ouverte de ces ordres de contrer le développement des établissements protestants. Pour les Sœurs, l'association entre action sanitaire et action éducative encourage certains habitants aisés d'une ville à majorité musulmane comme Naplouse (350 chrétiens pour plus de 30000 habitants musulmans et une petite communauté de Juifs samaritains) à leur confier l'éducation de leurs filles. Elles n'accueillent pas de garçons; c'est seulement sur les villes côtières et à Jérusalem que les Sours accueillent les jeunes garçons jusqu'à l'âge de 6 ans, avant leur entrée en école primaire chez les Frères des Ecoles chrétiennes généralement. On remarque d'ailleurs que pendant cette période, leur action sanitaire est très étendue dans le district, jusque dans des villages très reculés.

${ }_{134}$ Guide des missions catholiques, Sacrée Congrégation de la Propagande, 1936 ; cf. Les ouvres des Français à l'étranger, Annuaire, U. F. E., 1935.

${ }_{135}$ Cependant, l'analyse statistique de l'ensemble des corps professoraux montre une nette inflexion des nationaux français durant la période. L'ordre des Sœurs du Rosaire, indigène, issu de l'ordre des Sœurs de Saint Joseph a davantage recours à la langue arabe.
} 
observe durant la période une réflexion sur la langue, instrument de pouvoir ${ }^{136}$.

L'usage de cette langue pose aussi la question du rapport entre la langue et l'identité ${ }^{137}$. Elle apparaît également comme une langue refuge, un instrument identitaire, et le véhicule de pratiques linguistiques et culturelles spécifiques. Dans une Terre Sainte où tout se pense en termes confessionnels ${ }^{138}$, les relations de ce couple délicat « langue(s)-religion(s)» se déclinent en liens de domination, de diffusion, de cohabitation. La conjonction "et ${ }^{139}$ exclut ici une interprétation dans un sens de «langue religieuse ${ }^{140}$.

Le début des années 1920 est marqué par des symboles du lien entre pouvoir et religion catholique, en France (canonisation de Jeanne d'Arc le 16 mai 1920, achèvement des travaux et consécration de la basilique du Sacré-Cœur), mais aussi en Orient, avec la visite du cardinal Dubois, perçue comme une appropriation française du Levant. 1923 correspond également à la fin officielle du régime des capitulations ${ }^{141}$ et du français comme langue officielle.

Les rapports entre langues et religions font l'objet de débats entre les acteurs pendant la période. Cette période correspond en effet à la remise en cause radicale du protectorat catholique de la France en Palestine, traditionnellement au service de la langue française, transition

${ }^{136} \mathrm{Si}$ l'on ne peut véritablement mentionner de «politique linguistique » de la part des Britanniques, l'aménagement linguistique entrepris dès le début du Mandat change les rapports entre les langues, mettant à l'honneur l'arabe et l'hébreu, et à un niveau plus administratif, l'anglais.

137 Cf. KHALIDI, Rashid, Palestinian identity, the construction of a modern identity, New York: Columbia University Press, 1997.

${ }^{138}$ C'est d'ailleurs l'argument utilisé à maintes reprises par les défenseurs de l'enseignement confessionnel en Palestine, face aux tentatives de la Mission Laïque française (MLF). «Catholique et français vont de pair... la laïcité est proprement incompréhensible dans un Levant où confession et nationalité se confondent», CABANEL, Patrick, Une France en Méditerranée, écoles, langues et cultures françaises XIX $X^{e}$ XIX $X^{e}$ siècles, Grâne : Créaphis, 2006, p. 19.

${ }_{139}$ «Langue(s) et religion(s)».

${ }^{140} \mathrm{Au}$ sens de «langue des cérémonies religieuses ». Il n’y a pas de paroisse francophone à Jérusalem à cette époque.

${ }^{141}$ Le régime des capitulations, défini à l'époque de François I ${ }^{\mathrm{er}}$ par la Sublime Porte, consiste en des exemptions juridictionnelles et fiscales s'appliquant aux ressortissants «francs» des Echelles du Levant, muées au XIX ${ }^{\mathrm{e}}$ siècle pour des individus ou groupes communautaires, en un statut d'extraterritorialité, garanti par les consuls européens. 
sensible puisque la Palestine perd progressivement son statut de la «plus française des terres d'Orient». Tandis que l'anglais tente de devenir l'instrument par excellence de la «koine culturelle» de la Palestine du nouveau Mandat, le français semble conserver une place de choix parmi les communautés chrétiennes.

La période mandataire correspond de plus à un renforcement de la position centrale de Jérusalem. L'importance de la ville sainte, croissante sous la période des Tanzimat, réformes ottomanes (1856-78), se confirme par son statut de capitale pour l'ensemble de la Palestine; elle attire de ce fait davantage d'élites et de clientèles potentielles pour ces établissements ${ }^{142}$.

Cette étude s'articulera autour des problématiques de maintien et de diffusion de la langue française et de la religion catholique dans ces établissements, la langue française étant vecteur de religion catholique et la religion catholique vecteur de langue française ${ }^{143}$. A un moment où la remise en cause de la politique traditionnelle de la France au Levant envers les congrégations catholiques apparaît comme moins virulente, quelles sont les logiques de continuité et de rupture dans sa politique linguistique? Assiste-t-on à une remise en cause de la politique traditionnelle de la religion comme base pour répandre la langue ou au prolongement de ce soutien sous une autre forme?

Quels sont les bouleversements à l'intérieur et à l'extérieur de ces établissements provoqués par l'arrivée du Mandat anglais, officiellement protecteur de toutes les communautés religieuses et linguistiques de Palestine ? Ces établissements ont-il encore pour rôle de former de bons chrétiens ou sont-ils devenus des lieux de propagande civique? Sur quelle langue s'appuient-ils pour mener à bien leurs missions?

Seront d'abord analysées les tensions entre les différents partenaires de ces établissements religieux, et avec eux, les continuités et les ruptures de leur politique à l'égard de ces établissements, les injonctions des acteurs religieux à ces établissements, et les réponses qu'elles provoquent de la part des deux ordres, qui se perçoivent comme des véritables défenseurs de la langue française durant toute la durée du

\footnotetext{
${ }^{142}$ La période ne correspond pas à une logique d'expansion de ces ordres. Bien établis, ils tentent plutôt de maintenir leur influence durant le Mandat britannique.

${ }^{143}$ Elle mentionnera en contrepoint la place et le rôle des langues et des religions qui cohabitent avec le catholicisme et la langue française au sein de ces institutions.
} 
Mandat. Puis, seront analysés la place et le rôle accordés à la langue française et à la religion au sein des établissements, ainsi que les pratiques qui leur sont liées, notamment à travers la répartition des élèves selon leur communauté religieuse, le curriculum proposé, la place réservée à l'instruction religieuse, la langue de cette instruction, et les associations religieuses et culturelles dont sont membres les élèves.

\section{Des sources éparses}

Les correspondances entre les établissements, avec leurs hiérarchies respectives, La Propaganda Fide, le MAE, le Service des ouvres françaises à l'étranger et le poste consulaire de Jérusalem évoquent le repositionnement des autorités et des établissements dans la nouvelle configuration politique. La France a en effet perdu la bataille du Protectorat catholique qu'elle exerçait ${ }^{144}$ depuis le XVIII ${ }^{\mathrm{e}}$ siècle. Elles exposent également les budgets de ces ordres qui proviennent en partie du consulat de France, mais aussi de la Propaganda Fide et du Patriarcat latin et de l'œuvre d'Orient ${ }^{145}$. Auprès des autorités françaises, le progrès de la langue est davantage mis en avant que celui de l'évangélisation. La Maison Généralice des Frères à Rome détient de nombreux dossiers sur les fondations des établissements, leur fonctionnement, des rapports de visite, le Bulletin des Ecoles chrétiennes, certains manuels ainsi que des photos non disponibles auprès des communautés locales. Des rapports sur les intérêts français en Orient au début de la période donnent une idée de l'articulation des liens entre langue et religion. Il s'agit surtout du rapport du Frère Justinus (fascicule III, Section de l'enseignement du Congrès français de la Syrie (3, 4 et 5 janvier 1919) ${ }^{146}$. Certaines archives du PRO Public Record Office, mais également les Personnal Papers du ministre de

${ }^{144} C f$. NICAUlt, Catherine, «La fin du protectorat religieux de la France à Jérusalem (1918- 1924) », Bulletin du CRFJ, Centre de Recherche Français de Jérusalem, 1998, p. 7-23.

${ }^{145}$ L'analyse de certaines statistiques est délicate, les missions étant dans une logique de client vis à vis de la Propagande; d'autre part on remarque par la correspondance importante du Frère Evagre (le fondateur des collèges des Frères de Palestine) avec la Propagation de la Foi à Lyon, que le manque de budget pour étendre les œuvres est un problème chronique depuis la création des collèges en Palestine.

${ }^{146}$ Les autres rapports sont antérieurs, notamment le rapport Charlot (1906) et le rapport Pernot (1912). 
l'éducation britannique de Palestine, H. Bowman ${ }^{147}$, rendent compte indirectement de l'évolution des rapports entre langue et religion, notamment à titre comparatif au sein des organisations de jeunesse par rapport à la construction identitaire (l'enjeu de l'utilisation de la religion au sein des mouvements de jeunesse notamment).

Pour identifier ces relations, outre les archives diplomatiques ${ }^{148}$, les archives locales de ces communautés permettent d'aborder les réalités de ces liens et pas seulement les idéaux. Elles comportent également des distorsions ${ }^{149}$ et doivent donc être confrontées aux archives des différentes autorités romaines ${ }^{150}$. Les archives locales ${ }^{151}$ des établissements comportent les registres du personnel, les Historiques des écoles, documents dans lesquels sont consignés de façon parfois

\footnotetext{
${ }^{147}$ Personal Papers, archives du Saint Anthony's College, Oxford (non classés), et Bowman, H.E., Middle East Window, Londres : Longmans, 1942.

148 Outre les archives du Quai d'Orsay, 3 dossiers spécifiques, aux archives du MAE de Nantes, portent sur les Frères des Ecoles chrétiennes et sur les Sœurs de Saint Joseph de l'Apparition.

${ }^{149}$ Les statistiques internes de la communauté par exemple, très souvent, ne correspondent ni à ceux du Consulat, ni à ceux de l'administration britannique. Les communautés religieuses enseignantes ont tendance à ne pas fournir beaucoup de renseignements dans les questionnaires anglais qui servent à l'élaboration des statistiques officielles du Mandat, redoutant une trop grande ingérence de la part des Britanniques.

${ }^{150}$ Le tome de LETOUZEY \& ANE de 1923, consacré à la Congrégation des Scurs de Saint Joseph de l'Apparition (Paris) mentionne à plusieurs reprises l'établissement tenu par les Sœurs à Naplouse, et les actions sanitaires des Sœurs. Le Guide des missions catholiques, de la Sacrée Congrégation de la Propagande, de 1936, fait également un portrait rapide de ces deux ordres.

${ }_{151}$ Elles ont fait l'objet d'un projet de récolement, de classification et de sauvegarde (pour les deux communautés) mené avec le MAE, Quai d'Orsay (2006/ 2007), ainsi que d'un projet d'histoire orale auprès des anciens élèves. Elles sont également un des terrains du projet de recherche de l'ANR 2007 (CNRS) «Les fabriques de l'archive. "Faiseurs" d'histoire et "faiseurs" d'archives dans le Moyen-Orient contemporain » (coordonné par Christine Jüngen). On assiste à un processus de patrimonialisation depuis quelques années, à la projection des problématiques actuelles des communautés chrétiennes sur les périodes antérieures. D'autre part certains fonds ont été perdus en raison des événements politiques et de l'emplacement de certains de ces établissements près des zones de combat. Pour les Sœurs, il s'agit notamment des archives des communautés de Jérusalem, Bethléem, Ramallah, Beit Jala et Naplouse, archives rapatriées en partie en 1995, date à laquelle les Sœurs quittent définitivement la ville. Nous privilégions ici les établissements de l'intérieur de la Palestine mandataire.
} 
allusive mais souvent détaillée, les différents projets et activités, les activités de la communauté, les événements qui touchent de près ou de loin le collège. Ce qui est mentionné de façon régulière permet d'obtenir des informations sur la trame chronologique, de brosser un tableau des acteurs avec lesquels la communauté est directement en contact, les liens avec les autorités et traduit les préoccupations du moment, les enjeux identitaires, les obsessions sécuritaires, de la concurrence protestante et franciscaine.

Les stratégies des établissements sont parfois mouvantes en fonction de leurs interlocuteurs. Contrairement à d'autres fonds qui n'ont pas (ou peu) connu de problèmes de préservation, ni de classification ${ }^{152}$, ces deux ordres ont perdu de nombreux dossiers ${ }^{153}$. D'autre part, quelle place était laissée dans cet enseignement aux sentiments d'appartenance communautaire des élèves? De quelle nature étaient les solidarités établies avec le milieu des parents ? Quels sont les prismes déformant des mémoires de ces établissements avant 1948 ? Les minorités chrétiennes sont-elles abordées par le prisme de l'extranéité ? L'approche reste donc inachevée, car ces questions restent jusqu'à ce jour sans réponses. Les sources sont lacunaires sur les programmes complets de catéchisme, sur le programme de certains cours donnés en partie en langue arabe ${ }^{154}$, sur les méthodes d'apprentissage locales de la langue française qui, comme dans le cas des Franciscains qui ont repris une partie des ouvrages des Frères, s'appuyaient sans doute sur des

152 Collège de Terra Sancta des Franciscains. Les archives franciscaines, longtemps inaccessibles, le sont depuis quelques années. Ce collège a conservé l'ensemble des archives, y compris des manuels d'apprentissage dans un état de conservation remarquable; et celles-ci sont généralement bien classées (un frère franciscain, archiviste spécialisé, s'occupait toujours des archives, dans un local prévu à cet effet). Cela n'a pas été le cas pour les deux communautés enseignantes que nous analysons dans cette étude.

${ }^{153}$ Cette perte s'explique par les événements politiques qui ont affecté les écoles (guerres de 1948, de 1967 notamment), mais aussi par le fait que la direction des écoles n'a pas jugé utile parfois de conserver certains dossiers.

${ }^{154}$ Dans le cas du collège de Jérusalem, c'est le Frère Epiphane, libanais, qui est chargé durant toute la période des cours de catéchisme ( $c f$. fiches sur le personnel enseignant de la Maison Généralice). Comme ce dernier est de langue maternelle arabe, certains aspects du cours sont traités en arabe, mais le plus souvent en français. 
exemples religieux ${ }^{155}$. Enfin, la notion de prosélytisme n'est guère facile à aborder puisqu'elle implique de la part du converti un reniement envers sa société d'origine. Elle n'est que rarement explicitée dans les sources, par précaution autant pour ceux qui se convertissent que pour les Frères et les Sœurs.

\section{Des rapports au cour d'enjeux diplomatiques et religieux}

$\mathrm{Au}$ cours de cette période, deux sphères d'influence, anglophone et francophone, s'affrontent, l'une attache encore une importance au rôle de la religion comme vecteur de la langue jusqu'aux années 1930, tandis que la seconde propose une image détachée de cette situation traditionnelle. Jusqu'au début des années 1920 en effet :

catholique et Français sont synonymes. Tout ce qui contribue à conserver à la foi catholique sa vigueur concourt à maintenir la prépondérance de l'influence française. A l'inverse, tout ce qui porte atteinte à la foi catholique amène invariablement une diminution de cette même influence $[. .$.$] Le meilleur$ moyen de travailler à développer l'influence française, c'est de travailler à développer l'influence catholique ${ }^{156}$.

Les Frères des Ecoles chrétiennes et les Sœurs de Saint Joseph de l'Apparition, majoritaires au début du Mandat pour l'enseignement des filles et des garçons, continuent à se penser comme les seuls défenseurs de la langue française et donc à garder leurs modes de diffusion de la langue.

Les autorités françaises entendent continuer à accorder leur soutien aux ordres religieux. Pour les Frères et les Sœurs se pose alors la question de savoir comment rester loyaux envers la France tout en étant

155 Claude Langlois, dans son article «L'offre scolaire des congrégations féminines françaises dans le bassin méditerranéen oriental $\left(\mathrm{XIX}^{\mathrm{e}}\right.$ début du $\mathrm{XX}^{\mathrm{e}}$ siècles) » a soulevé à plusieurs reprises la difficulté d'aborder l'aspect de la mission éducative des Sœurs, in CABANEL, Patrick, Une France en Méditerranée, écoles, langues et cultures françaises $X I X^{e}, X X^{e}$ siècles, Grâne : Créaphis, 2006, p. 219.

${ }_{156}$ RIfFIER, Jean, Les cuvres françaises en Syrie (1860- 1923), Paris: L'Harmattan, 2000, p. 65. 
sommés par la puissance mandataire de poursuivre une reconnaissance des pouvoirs anglais et de ses implications dans le domaine linguistique.

Les buts avoués de ces enseignements confessionnels sont de former de « bons chrétiens » ${ }^{157}$, tout en basant tout l'apprentissage sur la langue française. Les marges de manœuvre sont souvent délicates ${ }^{158}$ avec les pouvoirs publics et les autorités ecclésiastiques impliqués dans ces liens entre langue et religion. Les modalités de ces relations politiques apparaissent dans différentes sources disponibles.

\section{Entre remise en cause du Protectorat français et « mission sacrée de civilisation ${ }^{159}$ » britannique}

Durant la période ottomane et encore pendant les premières années du Mandat, un large appel est lancé au français comme instrument de culture, selon une double logique : chez les non musulmans, le français est langue de protection et d'éducation, chez les musulmans, le français est indispensable pour faire carrière à l'intérieur du système ottoman rénové $^{160}$ car c'est la deuxième langue d'administration de l'empire. Les collèges des Frères sont alors réputés auprès des élites pour être

\footnotetext{
${ }^{157}$ Institut archéologique allemand, archives appartenant au fond des ACJ, 1905.

${ }^{158}$ Les établissements de ces deux communautés dépendent des subventions des autorités religieuses et consulaires.

${ }^{159}$ Selon le titre d'un ouvrage d'Henry Laurens, La question de Palestine, (tome deuxième), 1922-1947, une mission sacrée de civilisation, Paris : Fayard, 2002. Le terme a également été employé pour désigner l'action de la France au Levant, $c f$. BURRows, M., «Mission civilisatrice, French Cultural Policy in the Middle East, 1860-1914 », The Historical Journal, 29, I, 1986, p. 109-135.

${ }^{160} C f$. LAurens, Henry, Orientales III, «Le levant arabe à la fin de l'époque ottomane, 1880-1914 », CNRS : Paris, 2004. P. Dhorme, Prieur de l'Ecole biblique et archéologique de Jérusalem, La langue française en Palestine, 18 mai 1922 : «Bien que la langue officielle de l'administration [ fût] le turc et la langue des natifs l'arabe, le français langue des autorités, pour ceux qui appartiennent à la société éduquée et pour communiquer avec les étrangers, après la langue maternelle, le plus important était le français », Archives du MAE, Nantes, $n^{\circ} 172$.

Le collège de Jérusalem initie le mouvement d'expansion des Frères à toute la Palestine mandataire en l'espace de dix ans. Le présent article ne répondra pas à la question de savoir s'il y a "modèle», question moins pertinente dans le cas d'un établissement missionnaire que dans le cas d'un établissement créé par les pouvoirs publics français avec pour vocation de répandre un modèle éducatif (par exemple le lycée de Galatasaray à Istanbul ; $c f$. thèse de doctorat de Gulsun Guvenli, soutenue à l’EHESS en janvier 2007).
} 
multiethniques, multireligieux et pour mettre à l'honneur dans leurs programmes l'apprentissage des langues étrangères. Les établissements des Sœurs accueillent moins de populations étudiantes mixtes. Les Frères réputés pour leur succès dans les différents établissements en Egypte, s'implantent également avec une logique urbaine ${ }^{161}$ depuis le 15 octobre 1878, date de l'inauguration, sur un terrain offert par le Patriarcat latin. Au départ peu nombreux, ces établissements voient leurs personnels et leurs publics augmenter rapidement ${ }^{162}$.

En 1918, après des négociations dont la France sort perdante en Palestine, le consulat général de France à Jérusalem, abandonnant les velléités d'une souveraineté territoriale ${ }^{163}$, poursuit son soutien aux deux ordres éducatifs, ces établissements ayant été jusqu'alors des « fers de lance » de la présence française en Terre Sainte ${ }^{164}$.

Je saisis cette circonstance (6 000 francs accordés au collège pour faciliter sa réouverture) pour appeler votre attention sur l'urgence que présente l'envoi par vos soins en Palestine de missionnaires en nombre suffisant en vue de procéder sans délai à la réouverture de vos établissements [...] Le patriotisme et l'esprit d'abnégation dont les missionnaires français ont toujours fait preuve dans l'accomplissement de leur œuvre de propagande en Turquie et particulièrement en Palestine me donnent l'assurance que la gravité de cet état de choses ne vous échappera pas et que vous mettrez tout en œuvre pour assurer la réouverture dans les délais les plus brefs, des maisons d'instruction ${ }^{165}$.

161 Archives de la Maison Généralice, NH 815/1, correspondance du Frère Evagre.

${ }^{162} 5$ frères et 150 élèves en 1904 , date de l'ouverture de la section payante, également année où les Sœurs ouvrent leur école de Naplouse, et loi contre les établissements scolaires confessionnels en France.

${ }_{163}$ Cf. RIFFIER, Jean, Les æuvres françaises en Syrie, 1860-1925, Paris : L'Harmattan, 2000.

${ }^{164} C f$. CABANEL, Patrick, «Les deux vocations : catholique ou laïque ? La France, le Levant et l'islam, 1860-1920», in AUDISIO, G. (dir.) Religion et société, Aix en Provence : Publications de l'université de Provence, 1998, p. 199-209.

${ }^{165}$ Lettre du Comte de Chérisey, pour le ministre et par autorisation, chef du Bureau des écoles et des œuvres françaises à l'étranger, lettre du 8 janvier 1918, série E, Levant. 
En 1923, lors de la fin officielle des capitulations et du protectorat français non seulement sur les catholiques français mais aussi sur tous les catholiques, la Palestine perd progressivement son statut de « la plus française des terres d'Orient ». Pourtant, bien avant le début du Mandat britannique, les deux ordres éducatifs se sont implantés en Palestine en bénéficiant d'un réseau de soutien important en France ${ }^{166}$, la France qui reste attachée à la diffusion de la langue sur le mode qui a prévalu au $\mathrm{XIX}^{\mathrm{e}}$ siècle. La langue française et la religion catholique sont alors étroitement liées dans l'enseignement offert par ces établissements ${ }^{167}$ :

L'Angleterre n'était à peu près rien à Jérusalem, elle n'y avait pas de fonction de quelque importance, sa langue n'y était pas parlée, tandis que les œuvres les plus florissantes étaient françaises et qu'elles avaient répandu la langue française autant et plus même qu'en aucun coin de cet Orient que domine notre influence intellectuelle [...]. C'est sur la conservation des établissements des religieux français et du caractère résolument français de leur enseignement que nous devons concentrer nos efforts. C'est là qu'est le réduit de notre influence ${ }^{168}$.

Les Frères, et les Sœurs dans une moindre mesure, optent après la guerre pour faire de l'apprentissage de la langue française leur cheval de bataille, puis ils introduisent progressivement des langues étrangères tout en conservant des liens privilégiés avec les autorités françaises. Une nette différence doit être remarquée entre les établissements des Frères, autonomes vis à vis de la Custodie, et ceux des Sœurs, qui appelés par elle au $\mathrm{XIX}^{\mathrm{e}}$ siècle, continuent à être soutenus, financièrement et au niveau de leurs programmes et de leurs examens, par les Pères Franciscains (la Custodie) :

${ }^{166} C f$. NICAUlt, Catherine, «La fin du protectorat catholique de la France à Jérusalem, 1918-1924», Bulletin du CRFJ, 1999, p. 7-24.

${ }^{167} C f$. THOBIE, Jacques, «La France a-t-elle une politique culturelle dans l'empire ottoman à la veille de la première guerre mondiale ? » in La France et l'Est méditerranéen depuis 1850, Economie, finance, diplomatie, Istanbul : Analecta Isiana, Isis, 1993, p. 355 sq.

168 Robert de Caix au Ministre des affaires étrangères, La position de la France en Palestine, 28 octobre 1920, MAE, E 312/1/404. 
Nous sommes tous disposés à reprendre avec un nouveau zèle et le même dévouement que par le passé, nos œuvres de civilisation et de patriotisme ${ }^{169}$.

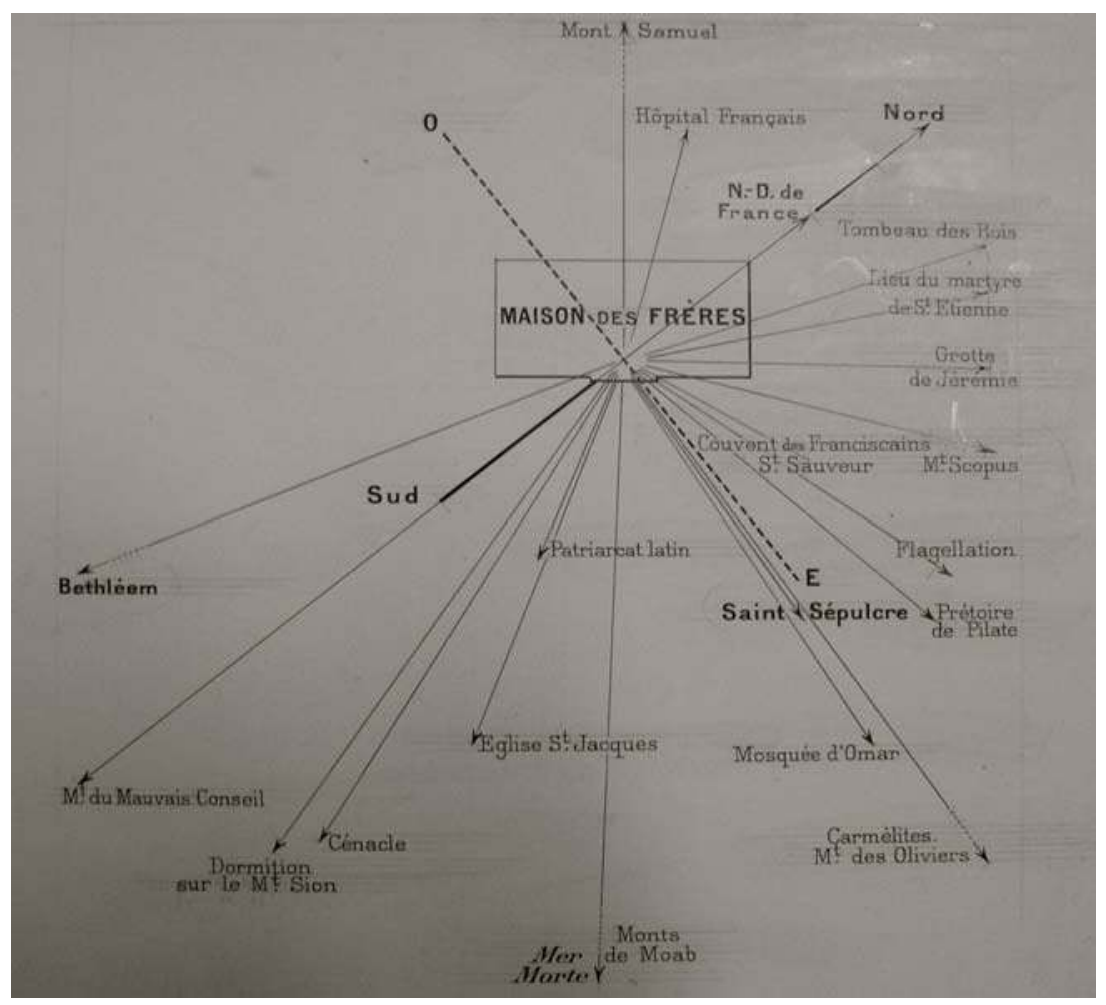

Position du collège des Frères par rapport aux autres institutions religieuses de Jérusalem (la centralité de l'établissement dans le réseau des ouvres françaises est souvent soulignée) - Archives du collège ${ }^{170}$

Les Frères sont souvent perçus comme d'abord animés par le patriotisme, avant que d'être des missionnaires :

${ }^{169}$ Lettre du Frère assistant au MAE, 28 décembre 1917, archives du MAE, Nantes, SOFE 200.

${ }^{170}$ Non daté mais appartenant à un dossier de l'époque mandataire. 
SE Le Cardinal Laurenti (...) me dit que d'après certaines informations qu'il avait reçues, le Frère visiteur de Jérusalem aurait commis dernièrement quelques imprudences de langage. A l'occasion d'une réunion qui s'est tenue à Jérusalem je crois, il a eu à prendre la parole et tout son discours aurait porté sur la mission patriotique des Frères au Levant, sans dire un seul mot du $1^{\text {er }}$ but que doivent se proposer les religieux dans les missions, qui est le bien des âmes. Il paraîtrait que ce silence à propos de l'apostolat religieux, et l'expansion de l'influence de la patrie terrestre donnée comme but unique ou du moins principal de l'œuvre des Frères, aurait très mal impressionné les assistants ${ }^{171}$.

Le consul G. Maugras lui-même déclare d'ailleurs aux Frères qu'il convient d' « éduquer de bons chrétiens qui connaissent la langue de leurs formateurs ». Dès 1919, le rapport du Frère Justinus souligne leur double inspiration, à travers "une politique d'expansion à l'étranger sous la double inspiration de la foi religieuse et du sentiment patriotique $^{172} »$. En 1927, le prieur des Dominicains, P. Dhorme, déclare se féliciter «du triple lien Patrie Education Religion qui garantit la pérennité de l'union que tant de causes tendent à dissoudre ${ }^{173}$ ».

La France assure ces ordres éducatifs de son soutien financier permanent, mais opère une sélection des établissements à favoriser ${ }^{174}$. Elle n'entend pas renforcer son soutien à la religion catholique mais au maintien de la langue, le couple « français / catholique » tend alors à se dissocier. Elle réoriente donc sa politique culturelle et linguistique et se tourne davantage vers le public juif, à partir de 1930.

Les Britanniques dissocient le couple langue / religion dès les premières législations. Dès 1922, le Education Department est créé au sein du Palestine Government. Les articles 15-16 du Mandat insistent d'ailleurs sur le devoir éducatif des autorités envers les populations

${ }^{171}$ Rome le 16 mai 1924, Frère Alexis Francois, au THF Allais Charles.

${ }^{172}$ Rapport du Frère Justinus sur les établissements des Frères au Proche-Orient, 1919, Chambre de commerce et d'industrie de Marseille. $C f$. également BARRES, Maurice, Enquête aux pays du Levant, tome 1, Paris : Plon, 1923, p. 193. Il est d'avis de donner "plus de poids » aux actions françaises, "plus spirituelles », qui doivent permettre de «s'attacher le cœur des Orientaux. Nous devons maintenir nos positions et par conséquent garder nos pièces maîtresses, à savoir nos congrégations enseignantes et charitables ».

173 Annales du collège, 1927.

${ }^{174}$ Dans les prévisions budgétaires des années 1929-1932. 
locales et le respect des minorités religieuses. La loi de 1924 stipule que la langue française n'est plus une langue officielle aux examens ${ }^{175}$, mais rien n'est stipulé sur la religion en tant que telle. Il en est de même pour les cursus et examens du Arab college, collège secondaire et Ecole normale pour instituteurs fondé dès 1918. La priorité est clairement l'apprentissage de la langue, chaque communauté est responsable de l'enseignement des religions.

En 1933, le Education Ordinance exige notamment des licences d'enseignement pour les professeurs, des inspections des agents du Education Department, et des formulaires annuels à renvoyer au haut-commissariat ${ }^{176}$. L'anglais devient ainsi la langue officielle de l'administration et sa maîtrise, de même que celle de l'arabe et de l'hébreu, garantit l'accès aux postes administratifs auxquels se destinent plusieurs élèves des Frères, tandis que le français reste la langue de distinction des catégories sociales les plus aisées. Ainsi son apprentissage ne diminue pas dans les emplois du temps pour filles durant cette période. Une des conséquences de ce nouveau système est l'inflation des matières profanes dans les programmes de ces établissements.

\section{Au cour de rivalités religieuses et linguistiques entre institutions locales et romaines}

Le contexte de concurrence dans lequel s'inscrivent les fondations de ces deux ordres est marqué par la division entre pouvoirs chrétiens et par des pressions, davantage sur les collèges des Frères que sur les établissements des Sœurs, de la part de la Propaganda Fide et du patriarcat latin. Les établissements sont soumis à la juridiction spirituelle $\mathrm{du}$ Patriarcat mais aussi à une certaine juridiction temporelle du Consulat Général de France à Jérusalem. Le Patriarche fait souvent le jeu de Rome contre les Frères, plus rarement contre les Sœurs ${ }^{177}$. Les

17526 avril 1924, MAE, E 312/ 32 «les langues d'examen (...) sont, à l'exclusion de toute autre, les trois langues officielles de la Palestine "anglais, arabe, hébreu" ".

176 TIBAwI, Abdul Latif, Arab Education in Mandatory Palestine : a story of three decades of British administration, Londres : Luzac, 1956.

${ }^{177}$ La brochure de la Custodie, imprimée par le Patriarcat et la Custodie pour le département palestinien de l'éducation en 1933 fait l'éloge de la plupart des écoles des Sœurs. Plusieurs rapports des consuls généraux de Jérusalem mentionnent l'attitude pro anglaise du patriarche, et pro italienne à travers le soutien apporté à la 
relations entre langue et religion sont perçues à travers le prisme du nationalisme par les institutions romaines. Ces deux ordres français ont été appelés par le Patriarcat au milieu du XIX ${ }^{\mathrm{e}}$ siècle, ils doivent œuvrer notamment à la «renaissance de l'orient chrétien ${ }^{178}$ ». Pourtant, plusieurs courriers reprochent aux Frères leur patriotisme et leur volonté de préserver l'usage du français avant celui de la religion, ou d'accorder l'exclusivité au français pour les cours de catéchisme, car les autorités estiment de manière classique que la langue est le moteur des pratiques religieuses.

Le règlement accordé aux établissements des Frères en 1891 leur redonne des droits que le Patriarche semble alors contester. Les Frères lui répondent alors :

Dans aucun cas nous ne pourrons porter ombrage à personne et moins encore constituer un danger de rivalité pour la grande famille franciscaine placée si fort au dessus de nous [...] Notre seule ambition, Très Saint Père, est de concourir, sous l'autorité des évêques et en qualité d'auxiliaires du clergé, à la formation chrétienne de la jeunesse [...] Notre mission en Palestine, Très Saint Père, est importante sinon nécessaire pour garantir la foi du peuple contre les envahisseurs des dissidents ${ }^{179}$.

En 1898, la Propaganda accuse à nouveau le fonctionnement des établissements des Frères par rapport à la langue française : selon la Propaganda, ils semblent faire moins de cas de l'enseignement religieux que de celui de la langue française. Elle critique également la présence des non catholiques, trop nombreux selon elle dans les établissements, et

Custodie. Bien que le patriarche ait fait au cardinal Dubois, en visite à Jérusalem en 1920 , des déclarations très favorables à l'influence française, il est soupçonné d'effectuer des démarches auprès des maisons religieuses non françaises pour qu'elles lui adressent directement leurs réclamations (à lui et non au consul de France) afin qu'il serve d'intermédiaire direct auprès des autorités britanniques. Il mentionne dès le début de son patriarcat la nécessité pour toutes les congrégations d'enseigner davantage en arabe, et dès le début du Mandat, en anglais également.

178 VERDEIL, Chantal, «Travailler à la renaissance de l'orient chrétien: les missions latines en Syrie (1830-1945) », Proche-Orient chrétien, 51, 2001, p. 267316 .

179 Lettre au Saint Père du Secrétaire de l'Institut des Frères des Ecoles chrétiennes au sujet des écoles de Terre Sainte. 


\section{Karène Sanchez-Summerer}

préconise une séparation physique entre les catholiques et les non catholiques $^{180}$ :

On dit que dans les écoles des Frères, on admet généralement dans une trop large mesure des enfants non catholiques et même juifs et cela avec peu de discernement (...) et puis la division entre catholiques et hétérodoxes tant recommandée par la Sainte Congrégation n'est presque pas observée. On dit que le but principal des écoles des Frères semble être celui d'enseigner la langue française, que les lettres et langues profanes s'y enseignent assez bien, mais que le catéchisme et l'histoire sacrée, qui devraient être l'objet principal, laisse beaucoup à désirer $(. .$.$) il se donne en langue française { }^{181}$.

Des pressions, qui n'aboutissent pas, sont parfois faites pour que les Sœurs de Saint Joseph cèdent l'enseignement du catéchisme, notamment aux Sœurs franciscaines qui veulent ouvrir une école paroissiale: «Elles alléguaient notre service à l'hôpital; si vous voulez nous ramasserons les enfants pour les préparer à leur première communion $^{182} »$.

Le débat autour de la langue d'enseignement du catéchisme est significatif. Dans une lettre de 1921, Monseigneur Barlassina, patriarche de Jérusalem, invite les Frères à ne pas se montrer aussi patriotiques « les missionnaires doivent se garder d'insister sur l'enseignement de la langue de leur pays, pour ne pas paraître plus préoccupés des intérêts de leur patrie que du salut des âmes ${ }^{183}$ ». Suivant les injonctions de la Propaganda Fide, "les missionnaires ne doivent pas se soucier des choses temporelles $[\ldots]$ ils éviteront de chercher à propager leur langue maternelle à l'étranger ». A plusieurs reprises, le Patriarche incrimine les Frères pour leur "inféodation" aux intérêts français. Face au phénomène progressif de l'arabisation dans les années 1930, le Patriarcat invite les Frères à améliorer leur enseignement de la religion et à dispenser les cours de catéchisme uniquement en arabe :

Il s'ensuit que tous les religieux et toutes les religieuses qui donnent une part secondaire à l'enseignement religieux et le

${ }^{180}$ Celle-ci ne sera jamais appliquée au sein des établissements.

${ }^{181}$ Propaganda Fide, Rome 13 mai 1898.

${ }_{182}$ Archives des Sœurs de saint Joseph de Jérusalem, historique.

${ }^{183}$ Circulaire de Mrg Barlassina, Patriarche de Jérusalem, 2 octobre 1920. 
distribuent de manière insuffisante se mettent en désaccord, non seulement avec la loi de l'Eglise, mais encore avec les intentions de leurs saints fondateurs et avec le but principal de leur Institut. L'étude de mémoire du texte du catéchisme est nécessaire pour retenir plus tard, les formules et les vérités principales, mais il est beaucoup plus important d'en comprendre le sens ${ }^{184}$.

Ceci est d'ailleurs une des conséquences de la promulgation de l'encyclique Orientalium Dignitas, datée du 30 novembre 1894, qui régit l'activité missionnaire au Proche-Orient et impose le respect des rites orientaux, ce qui implique que les langues locales soient remises progressivement à l'honneur dans les établissements des missionnaires dans le cadre de l'enseignement religieux.

Cela pose moins de problème aux Sœurs car, non seulement leur public est très majoritairement catholique, mais encore elles sont elles-mêmes à $60 \%$ en moyenne d'origine palestinienne ou libanaise ${ }^{185}$, ce qui n'est pas le cas des Frères qui restent à grande majorité de nationalité française durant la période. Ils ont donc davantage de difficultés à adhérer à ce projet missionnaire supranational, qui passe largement par l'enseignement de la religion en langue maternelle des élèves, c'est-à-dire en arabe.

L'enseignement de la religion doit être prioritaire. Aussi la méthode utilisée par les Frères dans les établissements toujours multiconfessionnels, est clairement critiquée :

Nous avons un auditoire mixte, composé de catholiques et de non catholiques; pour ne pas heurter ces derniers, il faut se limiter dans les explications ». Voulez-vous dire qu'il faut de la prudence dans les expressions pour ne pas heurter brusquement, tout en exposant la doctrine catholique dans son intégrité, et qu'il faut éviter les phrases hostiles, alors nous sommes parfaitement d'accord. Mais si vous dites qu'il faut passer sous silence tous les sujets qui plaisent peu aux non catholiques, ou cacher la vérité comme une pilule amère qu'on enveloppe sous une capsule pour la faire avaler, alors nous ne nous entendons plus. Est ce que par hasard vous

${ }^{184}$ Compte rendu général de l'examen de catéchisme dans les paroisses et les instituts religieux, année 1932

${ }^{185}$ Le terme d'« indigénisation » du personnel est généralement utilisé dans ce cas. 


\section{Karène Sanchez-Summerer}

refuseriez le pain aux enfants pour ne pas heurter les susceptibilités des étrangers? ou bien peut-être oubliez-vous votre beau titre de missionnaires. Ce titre vous savez, à l'occasion, le mettre en relief dans vos pays, mais ce titre et cette vocation vous imposent le devoir d'instruire ceux qui sont en dehors de la vraie foi ${ }^{186}$.

Le refus d'enseigner en arabe place les Frères dans une situation de désobéissance gravement préjudiciable pour l'ordre :

Désobéissance aux injonctions répétées de la SC de la Propagande qui a intimé l'ordre que l'Instruction religieuse soit donnée et étudiée dans la langue du pays. [...] «Nous n'avons pas de sujets pour enseigner en arabe ». Avant tout Nous répondons: ceci n'a rien à voir avec le texte du catéchisme, s'il ne s'y ajoute parfois les caprices d'une exagération politique de tout point lamentable [...] des vocations de langue arabe (sont reçues), mais ensuite (elles sont envoyées) en Europe [...] au lieu de faire fructifier cet important patrimoine de la langue qui devrait plus tard leur servir à faire tant de bien à leurs compatriotes. Quand comprendra-t-on qu'un religieux est un religieux, et non pas un représentant politique, et que ses devoirs sont sacrés et ne sauraient être mêlés à des visées purement humaines ${ }^{187}$ ?

Les Frères présentent l'inefficacité d'un enseignement qui serait donné en arabe dans les classes moyennes et supérieures, puisque le français est la langue courante de l'enseignement, et que les élèves la maîtrisent mieux que l'arabe. Ainsi, à leurs yeux, il ne s'agit pas seulement des rapports entre langue et religion, mais de toute l'organisation du système éducatif primaire et secondaire palestinien :

Les Frères expliquaient le catéchisme en langue française, langue courante dans nos établissements de Palestine, langue que les élèves connaissent parfaitement et comprennent souvent mieux que l'arabe littéraire, nécessaire pour une étude approfondie de la doctrine chrétienne. La leçon expliquée était étudiée dans les livres en langue française, permettant aussi au professeur de contrôler cette étude.

${ }^{186}$ Circulaire de Mgr Barlassina, Patriarche de Jérusalem, 2 octobre 1920.

${ }^{187}$ Idem. 
Pareil procédé est usité dans nos écoles et collèges d'Egypte, du Liban et de la Syrie, pays dans lesquels les délégués apostoliques n'ont qu'à se louer de la science catéchiste donnée par les Frères à leurs élèves, non seulement catholiques, mais encore schismatiques, musulmans et juifs, travaillant ainsi à étendre le règne du Christ. Désormais, pour obéir aux ordres formels de sa béatitude le Patriarche, les élèves ne pourront plus employer d'autre catéchisme que celui du diocèse en langue arabe, privant ainsi les professeurs de l'éminent moyen de contrôle qu'est la récitation du texte appris en rapport avec la leçon expliquée. Cet ordre formel est d'autant plus étonnant que 3 éminents prélats, Monseigneurs [sic] Bracco, Piavi et Camassei, qui se sont succédé sur le Siège patriarcal de 1873 à 1919, qui non seulement n'ont jamais rien trouvé à redire à l'enseignement religieux des frères FEC de Palestine, mais au contraire ont rendu les témoignages les plus élogieux sur les connaissances très étendues et approfondies en matière religieuse des Frères ${ }^{188}$.

Les rapports de visite exposent souvent l'inquiétude des visiteurs et indirectement celle des Frères envers les différents aspects de la vie religieuse des établissements, ainsi qu'une réflexion pédagogique sur cette langue d'enseignement religieux ${ }^{189}$.

${ }^{188}$ Réponse du Frère Visiteur à une lettre du Patriarche (septembre 1941) sur la défense d'enseigner le catéchisme en français.

${ }^{189}$ Certaines questions reviennent systématiquement « Les élèves se confessentils régulièrement? La communion est-elle fréquente ? Existe-t-il un patronage ? ». 


\section{Karène Sanchez-Summerer}

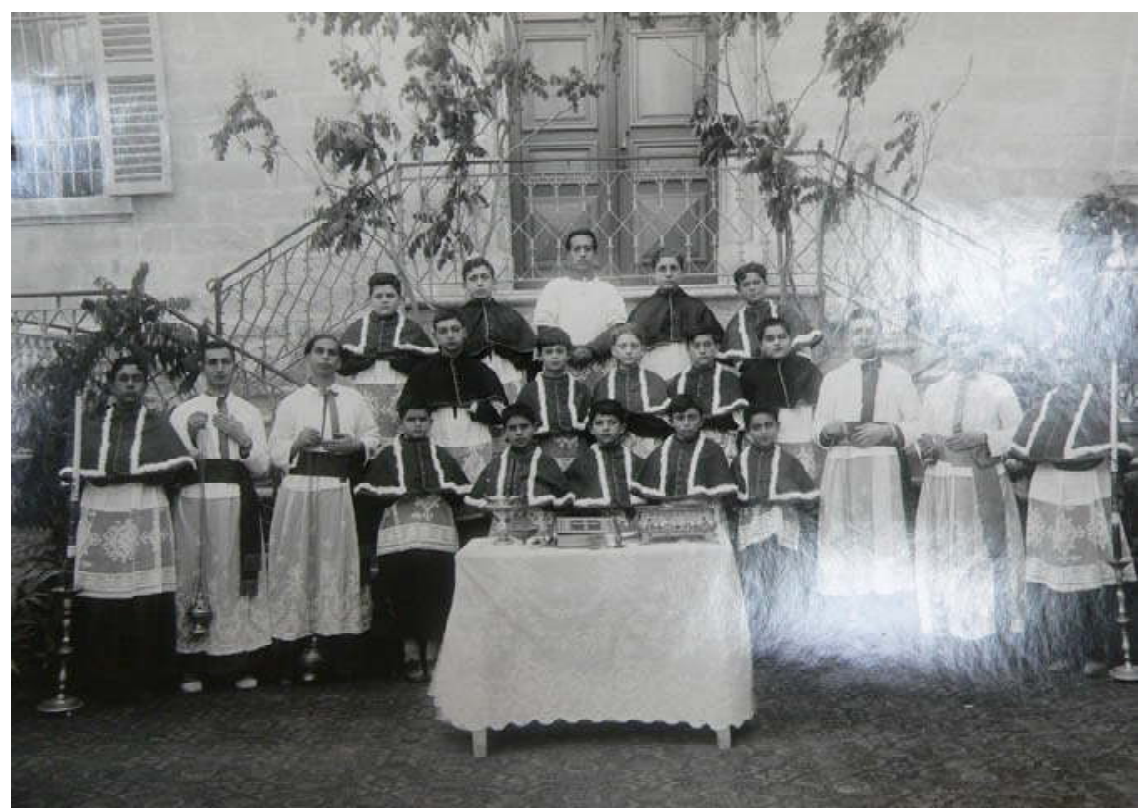

Célébration religieuse devant l'entrée principale du collège, soit lors de la fête du Sacré-Coeur, soit pour la Saint-Jean-Baptiste (fondateur de l'ordre) - Archives du collège -

Enfin, la «lutte» contre le protestantisme ou l'orthodoxie (plus que contre l'islam, sauf dans le cas particulier de Naplouse) est peu présente dans les établissements des Frères ${ }^{190}$; elle est plus marquée chez les Sœurs. Les conversions ou baptêmes sont davantage opérés par le biais de l'action sanitaire des Sœurs, au sein des dispensaires, et n'a que des liens très indirects avec la langue française (ils ne seront pas analysés ici). Au sein des établissements, il s'agit surtout d'influencer par l'enseignement de la langue française, la pratique catholique et

${ }^{190}$ Un seul cas de conversion est mentionné dans les annales du collège de Jérusalem; il ne fait pas l'objet d'un long développement; il s'agit d'un élève « israélite ». 
d'amener à «l'abjuration» les élèves «schismatiques ${ }^{191}$ ». Ainsi les Annales de Ramallah notent, dans le questionnaire sur le culte de Sainte Thérèse de l'Enfant Jésus du Patriarcat, que «la ville est infectée de sectes protestantes et celles-ci combattent énergiquement la lutte des saints. Malheureusement on en sent l'influence ${ }^{192} \gg$.

Il s'agit aussi de maintenir au sein du catholicisme les élèves, et d'éviter ainsi de manière indirecte les mariages mixtes entre chrétiens. Ainsi à Ramallah en 1927, les Sœurs remarquent au sujet du refus d'une de leurs élèves de se marier avec un orthodoxe à qui elle avait été promise, «les mariages mixtes deviennent la calamité du pays; en se mariant la jeune fille doit embrasser la religion du pays ", à majorité orthodoxe. En 1928, elles remarquent :

les Enfants de Marie qui ont été admises à la Congrégation se sont mariées avec des catholiques, tandis que celles qui n'ont pas voulu s'enrôler sous l'étendard de Marie n'ont pas eu la même chance, et ont embrassé (la) religion (de leurs époux ${ }^{193}$ ).

\section{Stratégies pragmatiques au sein des établissements}

Les Frères sont parfois perçus par les autorités ecclésiastiques, voire par les consuls français, comme des promoteurs d'un idéal d'enseignement qui n'est pas opposé à l'idéal de certains établissements laïcs, dans leur transmission du modèle culturel français, leur rôle pour les formations

191 A Ramallah par exemple, juste avant la première guerre mondiale, les Annales font état de la conversion d'une élève protestante qui refuse d'assister à des cours de catéchisme catholique; elle déclare en effet vouloir suivre uniquement des cours de français chez les Sœurs. Les annales mentionnent qu'elle est « touchée » par le catholicisme grâce à la méthode de français des Sœurs qui utilisent essentiellement des textes catholiques pour l'apprentissage. « Dès qu'elle commença à lire, nous lui faisions lire le souvenez-vous à Saint Joseph sur un petit livre en guise de leçon ».

${ }^{192}$ Les cas de conversions sont rares. Certaines familles arméniennes se convertissent, ou bien il s'agit d'orphelines qui rentrent ensuite dans l'ordre, $c f$. Annales de l'école de Bethléem, conversion de 3 élèves arméniennes orthodoxes, lettre du 25 mars 1927, "les enfants et leurs parents se sont convertis non pas à l'église arménienne catholique mais bien à l'église latine ».

${ }^{193}$ Beaucoup d'entre eux appartiennent à la communauté orthodoxe. 
professionnelles dispensées (réputées assurer l'insertion professionnelle des élèves dans toute la région), et leur vision de la promotion de la langue française. Ils doivent composer avec les attentes des parents, celles du Consulat, du ministère de l'Education britannique palestinien pour qui l'enseignement de la religion est détaché de celui de la langue.

\section{Un recrutement diversifié}

Les catégories d'élèves sont plurielles, ethniquement, religieusement et sociologiquement. Chez les Frères, la majorité d'entre eux sont catholiques sur l'ensemble de la période $(50 \%$ à $65 \%$ des élèves des collèges des Frères $\left.{ }^{194}\right)$, d'autres communautés chrétiennes sont présentes (notamment des orthodoxes, des protestants et des arméniens), ainsi que des musulmans et des Juifs ${ }^{195}$ (de 8 à $11 \%$ des élèves), mais peu d'Européens (au maximum une vingtaine sur un total de 200 à 250 élèves du collège; aucun en école gratuite ${ }^{196}$ ). Hormis les périodes de tension politique aiguë dans la vieille ville (1928-29, 1937-39), le recrutement est constant dans les collèges et écoles gratuites des Frères.

${ }^{194}$ Statistiques des archives locales des communautés, ainsi que celles des BB, Blue Books britanniques.

${ }^{195}$ Les Frères accueillent rapidement des élèves juifs. Issus en général de familles aisées, ceux-ci participent au système de subventions pour les écoles gratuites des Frères. Aucun de ces éléves n'a été recensé par les Frères comme s'étant converti au catholicisme.

${ }^{196}$ Blue Book, Education Department, Annual Reports; statistiques fournies au consulat chaque année, Annales du collège et MAE, Nantes, Fonds des Euvres. 


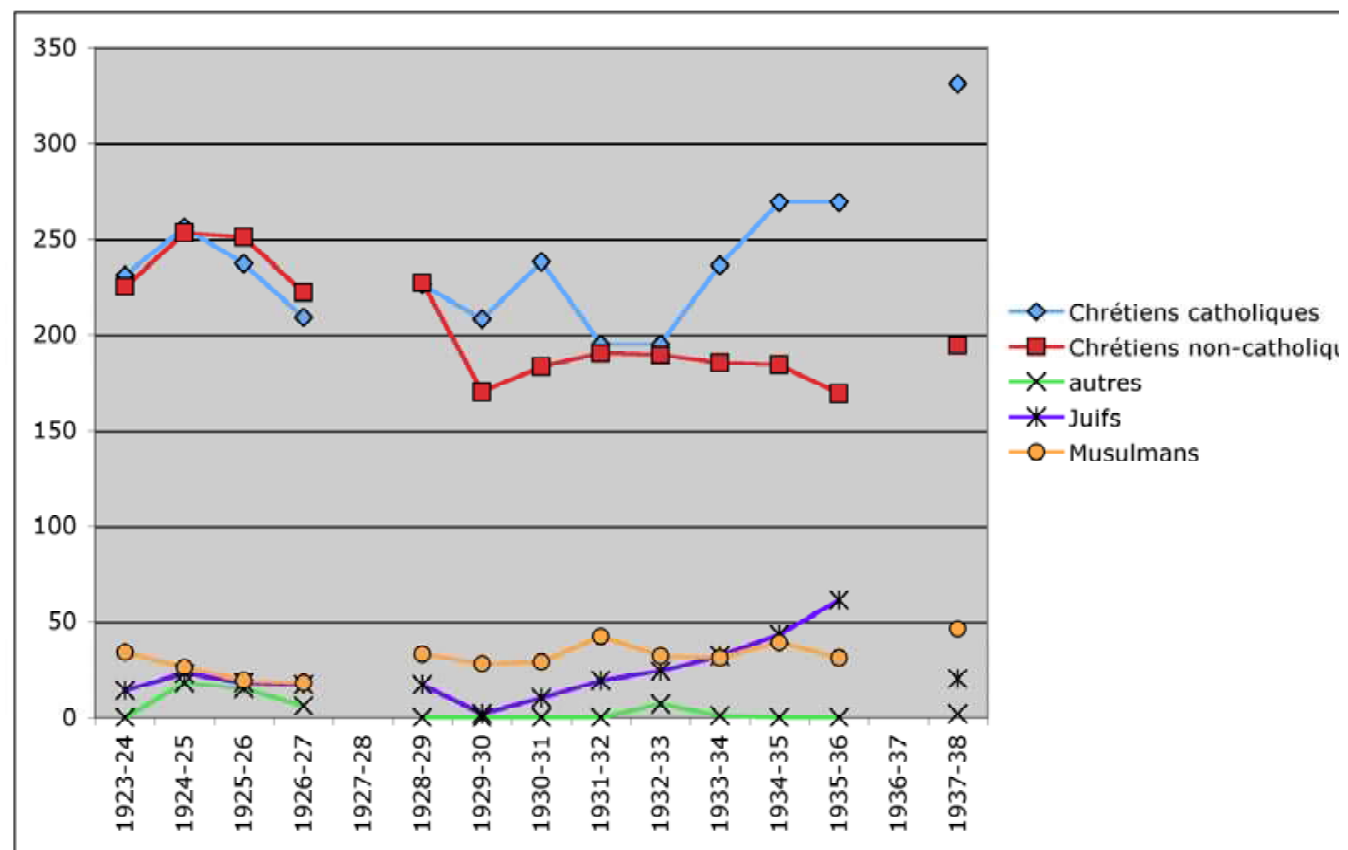

Elèves par religion, Collège des Frères de Jérusalem (1923-1937)

- D'après les archives du collège -

Progressivement sur la période un changement sociologique peut être observé, des classes moyennes, catholiques et non catholiques inscrivent également leurs enfants dans ces collèges, davantage attirés par la formation professionnelle et l'apprentissage de trois langues étrangères (français, arabe et anglais). Ils semblent alors trouver dans l'éducation française un moyen d'ascension sociale ; la nature de ces établissements, à la réputation de tolérance à l'égard des autres confessions, ne freine donc pas l'inscription des non catholiques. La langue française apparaît comme la langue des minorités chrétiennes et des classes moyennes, davantage que comme langue de l'éducation exclusivement catholique. 


\section{Karène Sanchez-Summerer}

Plusieurs parents sollicitent les Frères pour augmenter le nombre d'heures de cours en arabe ${ }^{197}$.

Le public des écoles des Sœurs est en général plus homogène (grande majorité d'élèves catholiques, une ouverture discrète aux élèves musulmanes à partir des années 1930). Les années 1920 correspondent en effet à une demande éducative plus forte des filles. L'école de Jérusalem est celle qui accueille le plus de filles chrétiennes non catholiques (de 10 à $25 \%$ sur la période). Au $1^{\mathrm{er}}$ janvier 1918, 385 élèves sont inscrites pour la réouverture. A la fin des années 1930, plus de 620 filles suivent l'enseignement des Sœurs. L'action éducative des Sœurs est toujours associée à leur action sanitaire (elles dirigent généralement des hôpitaux et effectuent des visites à domicile auprès des malades, même dans des villages reculés). L'association de ces deux actions favorise la confiance de certaines communautés non catholiques qui confient alors l'éducation de leurs filles aux Sœurs, tandis que les garçons sont scolarisés dans des établissements communautaires.

Le cas de l'établissement de Naplouse est exceptionnel car, dans une ville presque monoconfessionnelle musulmane, certaines familles d'élites sollicitent une éducation en français pour leurs filles ${ }^{198}$ :

Mais était-ce assez de s'occuper des corps? Les religieuses pensaient avec raison qu'un autre champ plus vaste s'offrait à leur zèle, elles voulurent atteindre les âmes, et pour ce faire, elles comprirent que l'unique moyen était l'école. Du reste elles y avaient été sollicitées par les bonnes familles de Naplouse désireuses de procurer à leurs filles les mêmes avantages que trouvaient celles d'autres villes. Il y avait bien à Naplouse une petite école paroissiale, tenue par les très dignes religieuses du Rosaire pour les chrétiennes, mais pour les musulmanes rien n'avait été prévu ${ }^{199}$.

\footnotetext{
${ }^{197}$ Les raisons invoquées sont d'ordre plutôt identitaire : les parents soulignent l'importance pour leurs enfants, en tant que «Palestiniens » (l'adjectif " arabe » n'est pas employé) de maîtriser davantage l'arabe ; $c f$. correspondance avec les parents, archives du collège des Frères de Jérusalem.

${ }_{198}$ Dans son ouvrage de 1927, Naplouse et son district, le père Jaussen de l'Ecole biblique et archéologique de Jérusalem livre différentes anecdotes sur la réception par les familles musulmanes de l'éducation des Sœurs, leur bienveillance à l'égard des élèves, et les accusations qui portent sur ces «filles de la montagne, banat al jabal », lorsque des élèves réagissent de manière trop « émancipée » selon leurs familles.

${ }^{199}$ Historique de la communauté de Naplouse, note sur la création de l'école.
} 
Cependant, tant dans l'organisation des cursus offerts que dans leur approche, les Sœurs établissent tout au long de la période des liens plus forts que les Frères entre langue française et religion catholique, ainsi :

Les élèves pour la plupart musulmans profitent à cette école de vertu, leurs mœurs s'améliorent, leur conduite s'assagit et beaucoup d'habitudes plutôt blâmables disparaissent (...) et que de préjugés à l'égard de notre religion qui tombent, que de sympathie dans les cœurs pour la croyance des Infidèles, hier encore objet d'exécration aujourd'hui tolérée avec bienveillance, demain aimée et recherchée ${ }^{200}$.

\section{Curriculum, « Des âmes à conquérir, des intelligences à éclairer $^{201}$ »}

On observe pour la période un basculement du français à l'arabe pour l'enseignement du catéchisme, bien que les rapports des Frères visiteurs mentionnent jusqu'en 1939 l'insuffisante connaissance de la langue maternelle des élèves par les Frères. Si le rôle des établissements est réaffirmé quant à la "formation de bons chrétiens », la lourdeur des cursus et la concurrence éducative poussent les établissements pour garçons, davantage que ceux pour filles, à mettre l'accent sur les matières profanes, et à adopter une «stratégie défensive ${ }^{202}$ » minimale quant à l'éducation religieuse, surtout destinée aux catholiques palestiniens. D'après les différentes archives, on ne peut guère parler de volonté de latinisation des élèves non catholiques, au sein des établissements des Frères. Le français n'est pas d'emblée perçu comme la langue de diffusion de la religion catholique. Il n'y a pas de mise en péril de l'identité religieuse des non catholiques car la religion est souvent perçue comme une matière comme les autres ${ }^{203}$ ( $c f$. Prospectus du collège de Jérusalem, voir ci-dessous p.132).

${ }^{200}$ Historique de la communauté de Naplouse.

${ }^{201}$ BARRES, Maurice, Enquête aux pays du Levant, tome 1, Paris : Plon, 1923.

202 RIFFIER, Jean, Les cuvres françaises en Syrie (1860- 1923), Paris : L'Harmattan, 2000.

${ }^{203} \mathrm{Ceci}$ explique aussi sans doute la raison pour laquelle au collège de Jérusalem de nombreux élèves juifs sont souvent premiers de classe en catéchisme. Dans l'obtention de prix et des diplômes la religion semble aussi une matière comme les autres. 
Pour s'adapter à la demande sociale (une révision et une adaptation des programmes est souhaitée par les parents et les autorités), garder des élites et sauvegarder la réputation du collège, les Frères mettent l'accent sur l'apprentissage des langues étrangères (les Sœurs également, mais dans une moindre mesure) ce qui alourdit de manière significative les matières profanes. Ils tentent aussi de revaloriser la langue arabe. La concurrence scolaire diversifie en effet les offres linguistiques dans ces établissements pour garçons et pour filles. En 1934 est même introduit dans le cursus l'apprentissage de l'hébreu ${ }^{204}$. Les établissements des Frères sont d'ailleurs comparés à certains établissements laïques, même si leurs résultats aux examens français baissent sur la période ${ }^{205}$, d'où l'échec de la mission laïque à Jérusalem en vue d'établir un lycée ${ }^{206}$. D'après quelques éléments d'un corpus, il est également significatif que les Frères ne s'appuient pas, contrairement aux Franciscains dans leurs écoles primaires, sur des exemples religieux $^{207}$.

La formation religieuse des élèves catholiques consiste en prières scandant la journée, catéchisme, assistance à la messe les jours de classe et les dimanches ou jours de fête, offices de la chapelle (pour tous les élèves chrétiens, les musulmans et les Israélites sont pendant ce temps en salle d'étude), retraites annuelles du collège. L'éducation religieuse n'a finalement pas assez de poids pour remettre en question

${ }^{204}$ Il ne s'agit dans ce cas ni d'une prise en considération de la langue maternelle des élèves (les élèves juifs sont à cette époque peu nombreux au collège), ni de la religion juive mais plutôt d'un souci de débouché professionnel pour les élèves. Les Frères estiment en effet que la maîtrise de l'hébreu permettra à leurs élèves de postuler à des postes de l'administration mandataire, puisque l'hébreu est une des trois langues officielles du Mandat. Le cours est rapidement abandonné, faute d'un nombre suffisant de participants.

${ }^{205}$ D'où la fin de l'équivalence avec le baccalauréat français en 1931. MAE, SOFE, $\mathrm{n}^{\circ} 200$, correspondance du vicomte d'Aumale sur le diplôme offert par les Frères en Palestine.

${ }^{206}$ Le résultat de la mission de la MLF est l'instauration d'un centre de culture française, le CCF, en 1937, dans un lieu proche du Consulat lui-même, à l'ouest de la ville, qui ne réussira jamais vraiment à rassembler les intelligentsia arabes (musulmane et chrétienne) et juive.

${ }^{207}$ Institut archéologique allemand, notes sur le collège des Frères de Jérusalem et les écoles de la custodie, varia. Les Franciscains prennent des exemples dans les missels pour l'apprentissage de l'arabe, plus important en volume horaire que chez les Frères. 
l'appartenance à la communauté religieuse d'origine des différents élèves. Pour les établissements des Sœurs, le curriculum est moins complet « on y enseigne la langue française, l'arabe, l'anglais et l'ouvrage manuel (couture broderie, tricot) ${ }^{208} »$, l'enseignement des langues étrangères y est important « 3,5 heures de français par jour, une heure d'arabe ainsi qu'une heure d'étude, une heure d'anglais et une heure d'étude ». La religion y occupe une place plus importante. Ainsi, à Jérusalem, 16 filles se présentent au concours de catéchisme en 1926; elles sont plus de 30 dans les années 1930. L’homogénéité plus importante du public entraîne moins de remarques de la part des parents et du Patriarcat qui, tout au long de la période, félicite les Sœurs pour leur enseignement du catéchisme, en français et en arabe. Les parents semblent davantage favorables à l'apprentissage du français.

Les remarques des Frères Visiteurs lors de leurs rapports annuels et celles de l'Institut des Frères des Ecoles chrétiennes sur l'importance de la rénovation de l'enseignement du catéchisme et de la langue dans laquelle il devrait être enseigné indiquent les insuffisances de cet enseignement dans les cursus proposés ${ }^{209}$. Le manque de piété de la part des élèves et l'absence de certaines pratiques religieuses comme les prises de communion (collèges payants et écoles gratuites) est relevé par l'ensemble des Frères Visiteurs pour la période mandataire ${ }^{210}$. A partir du début des années 1930, chaque langue (français, arabe et anglais) fait l'objet de remarques différentes sur l'évolution de son enseignement et de sa maîtrise par les élèves et les maîtres (différences entre les collèges et les écoles gratuites, où les lacunes semblent être plus significatives). En 1932, le catéchisme est mentionné comme étant enseigné en arabe par le Frère Visiteur Philotée. En 1933, le Frère Visiteur Oger déplore que les élèves s'éloignent des sacrements :

1936 - collège: d'année en année le français perd de son influence (...) trop d'étrangers dans le corps enseignant et les

${ }^{208}$ Historique de la communauté, Ramallah, archives de la communauté, 1924.

${ }^{209}$ Aux chapitres généraux de 1928 et 1934 de l'Institut il est peu question de ce sujet, mais l'Institut publie notamment le manuel du catéchiste en 1907. Pas de rapports pour les Sœurs signalant des lacunes de ce point de vue, mais la nécessité de recourir parfois à l'arabe, langue maternelle des apprenantes; les établissements ne possèdent pas encore assez de sœurs « indigènes ».

${ }^{210} \mathrm{Ne}$ sont évoquées ici que les remarques concernant les collèges payants et les écoles gratuites, pas celles qui touchent aux communautés des Frères. 
familles le réclament moins, la préparation au Matriculation semble primer sur tout. Mais donnera-t-il le résultat qu'on attend?

1936- école du Sacré Cœur: l'anglais et l'arabe semblent mieux suivis et contrôlés. Pas suffisamment de piété, pas de réunions de catholiques ni de congréganistes ${ }^{211}$.

Certains Frères s'appuient sur la mixité de leur public et leur longue expérience de la coexistence comme forme de «témoignage catholique » auprès des élèves non catholiques pour atténuer certains éléments du catéchisme ${ }^{212}$. Tout au long de la période, le Patriarcat reproche aux Frères l'insuffisance de l'enseignement de la religion dans les programmes et l'absence de liens entre les établissements des Frères et la paroisse, les prêtres du Patriarcat latin :

Un pareil désordre habituel en s'approchant de la confession indique l'absence d'esprit religieux; ce que d'ailleurs nous constatons péniblement dans l'école des Frères à l'occasion de la visite que l'inspecteur de religion fait chaque année [...] d'ailleurs depuis combien d'années et combien de fois, n'ai-je pas exprimé mon mécontentement pour l'insuffisance de la partie religieuse dans vos écoles de Palestine. Exception faite pour Haifa [...] dans les autres écoles payantes du diocèse les Frères se laissent dépasser par toutes les communautés. L'influence du prêtre, à qui Dieu a directement confié le salut des âmes, est complètement écartée, et le cachet de piété, qui devrait être foncièrement gravé dans les élèves d'une école catholique, manque dans les vôtres. Je regrette de le dire, vous soignez surtout l'instruction, mais la formation religieuse vient en dernier lieu ${ }^{213}$.

${ }^{211}$ Rapport de visite, Maison de Jérusalem, Archives de la Maison Généralice de Rome, 1936. Mêmes remarques jusqu'en 1939, avec des nuances positives sur la piété pour cette année-là.

${ }^{212}$ Le 20 octobre 1926 par exemple, le directeur du collège de Bethléem écrit au secrétaire général des Frères sur le cours d'histoire sainte, pour indiquer qu'il n'est pas possible de faire cours avec ce type de manuels dans des classes mixtes; la réaction du petit noviciat de Bethléem en 1927 est contradictoire, la décision est prise de ne plus enseigner l'anglais «pour ne pas perdre l'influence du catholicisme ».

${ }^{213}$ Le 9 octobre 1940, extrait de la lettre du patriarche Barlassina au Frère Directeur de Jérusalem. 
Les lacunes des Frères, passeurs de religion et passeurs de langue sont soulignées pour la langue arabe et parfois, celles des enseignants «civils» pour la religion. Ces remarques portent également sur les prêtres du patriarcat et sur la pédagogie même de leur enseignement religieux :

1938: école gratuite: comme au collège l'enseignement religieux laisse un peu à désirer, car les Frères ne connaissent pas suffisamment l'arabe, langue maternelle (...) les civils et les prêtres indigènes ne savent pas enseigner le catéchisme.

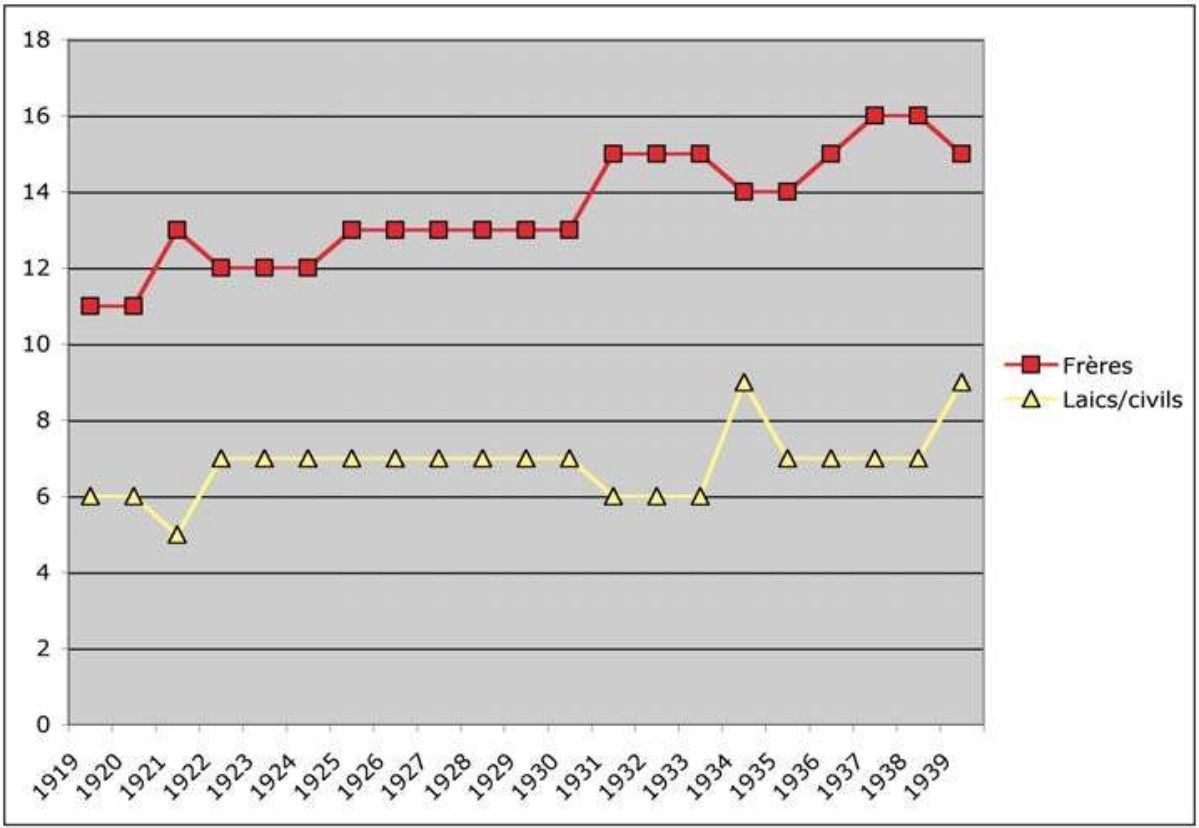

Répartition du corps professoral, collège des Frères de Jérusalem (19191939) - D'après les archives du collège -

Pourtant les rapports des Visiteurs et l'Institut des Frères mettent en lumière l'importance du sacerdoce pour le catéchisme enseigné par les 
Frères. Une circulaire du Frère Supérieur Junien Victor de 1938 est consacrée exclusivement à « l'action catéchistique des Frères des Ecoles chrétiennes »; elle rappelle que la fondation de l'Institut s'est inscrite dans « un vaste mouvement d'apostolat vers l'enseignement élémentaire de la religion [...] la vocation catéchiste dévolue aux Frères est plus nécessaire que jamais » (p. 24 de la circulaire). Cette circulaire évoque ensuite des aspects de la méthodologie de l'enseignement de la religion en français. La formation des enseignants est évoquée pour souligner le relâchement des liens entre l'enseignement de la langue et de la religion. Contrairement à ce qui se passe chez les Sœurs (davantage de sœurs palestiniennes ou libanaises ${ }^{214}$ ), il n'y a guère de processus «d'indigénisation ${ }^{215}$ » des Frères. Les maîtres civils sont peu formés au niveau religieux ${ }^{216}$ (s'ils connaissent l'arabe il leur est généralement reproché de ne pas savoir enseigner la religion). Depuis 1894, l'école de formation à Bethléem ${ }^{217}$ ne suffit guère aux besoins, et les novices sont envoyés en France et parallèlement les maîtres civils augmentent au sein de l'Institution. La complexité des programmes soumis à l'inflation de l'apprentissage de langues étrangères est parfois déplorée car elle entrâne une négligence des cours de religion: «cela vient de la complexité des programmes qui rend impossible l'enseignement religieux à une même heure dans toutes les classes ${ }^{218}$ ».

Certains Frères pensent que la langue arabe insuffisamment maîtrisée par les adolescents, ne peut leur permettre de penser les concepts véhiculés dans les cours de catéchisme, contrairement à la langue française qu'ils maîtrisent davantage.

Pour les autorités romaines et le Patriarcat, il s'agit de mettre l'accent sur l'arabe dans le programme des cours de religion et des

${ }^{214}$ Les Sœurs du Rosaire, issues de l'ordre des Sœurs de Saint Joseph, sont, elles, toutes indigènes et enseignent le catéchisme ainsi que la religion systématiquement en arabe. Les sœurs sont palestiniennes ou parfois libanaises durant cette période.

${ }^{215}$ Augmentation du nombre de frères palestiniens parmi les Frères.

${ }^{216}$ Ils n'est pas nécessaire pour être enseignant dans ces établissements de suivre des séminaires religieux.

${ }^{217}$ Il est à noter que dès le départ, « la création d'une Ecole Normale pour Instituteurs n'aboutit pas, [...] les sentiments du Patriarche (italien) à l'égard des Frères ayant changé ", Archives de la Maison Généralice, Rome, dossier Bethléem NH 851/1, et BEDEL, Henri, Etudes lassaliennes 11, p. 106.

${ }^{218}$ Rapport de visite, Frère Palatin, Maison de Jérusalem, Archives de la Maison Généralice de Rome, 1938. 
matières profanes, car la langue a des implications importantes sur l'identité palestinienne en formation:

1- jusqu'ici les œuvres scolaires catholiques dans le ProcheOrient ont dû leur prospérité au soin qu'ont apporté les missionnaires de s'adapter aux besoins régionaux.

2- mais pour ce faire les missionnaires ont imposé leur propre langue nationale à leurs disciples. Conséquence: la plus grande partie du personnel de ces auvres ignore la langue arabe (...).

3- le nationalisme régional exige actuellement la réhabilitation de la langue arabe. Avant que les exigences officielles $[\ldots]$ ne restreignent la liberté de l'enseignement et n'imposent des programmes auxquels les religieux ne sont pas préparés, il est de toute nécessité que les Supérieurs préparent des éléments capables de faire face à la nouvelle situation ${ }^{219}$

Contrairement aux affirmations de Barrès ${ }^{220}$, peu d'âmes sont conquises au catholicisme à la fin de la période mandataire, mais beaucoup le sont à la langue et la culture française diffusées par ces deux ordres religieux. Tout en répondant à la demande d'arabisation des programmes émanant de leurs clientèles ${ }^{221}$ et des autorités religieuses, par un enseignement aux programmes laïques (faible poids de la religion dans les programmes et les examens, et similitudes avec les programmes laïques de primaire supérieur de la métropole), ces établissements choisissent donc de ne pas enseigner le français, comme un simple outil de communication (c'est le cas de l'anglais) mais d'enseigner en français ${ }^{222}$.

${ }^{219}$ La Sainte Congrégation pour l'Eglise Orientale, circulaire « aux maisons généralices et à la direction des œuvres scolaires dans le Proche-Orient arabe ».

220 «Des âmes à conquérir, des intelligences à éclairer», BARRES, Maurice, Enquête aux pays du Levant, op.cit., 1923.

${ }^{221}$ Celle des écoles des Frères bien plus que celle des Sœurs.

${ }^{222} C f$. LAFON, Jacques, "Langue et pouvoir; aux origines de l'exception culturelle française », Revue Historique 592, 1994, p. 418. 


\section{Des associations pour la promotion de la langue française et de la religion catholique?}

Certaines associations promeuvent la religion comme vecteur de langue française, même si leur rayon d'action diminue face à l'encadrement de la jeunesse proposé par les Britanniques et qui ne met pas au premier plan l'affirmation d'une éducation religieuse par une langue spécifique. Il s'agit notamment de la YMCA Young Men Christians Association (établie en 1876, son nouveau bâtiment imposant en face du King David Hôtel, symbole du pouvoir britannique, est inauguré en 1933).

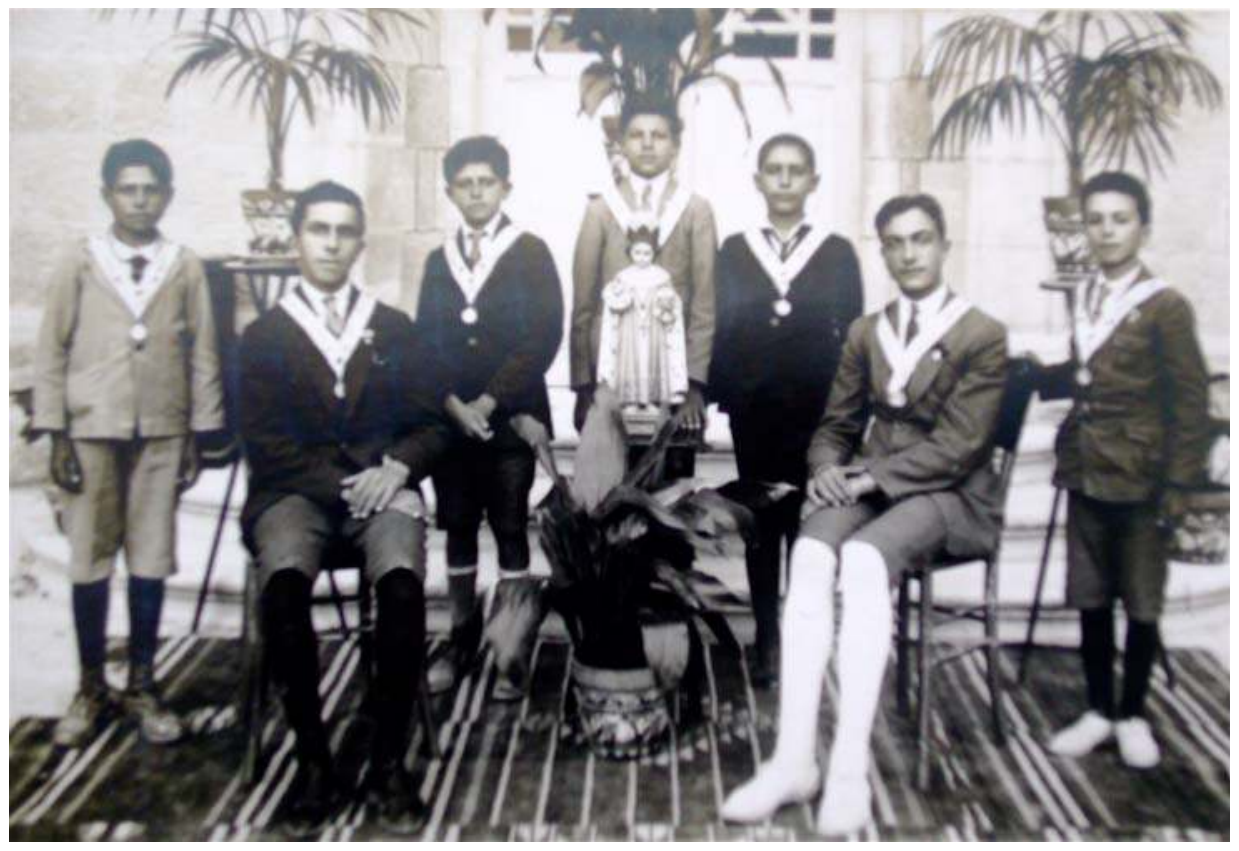

Membres de l'archiconfrérie du Très Saint Enfant Christ (fondée en 1910), collège de Jérusalem - Archives du collège -

La plupart de ces associations sont créées à la fin du XIX siècle. Les membres des confréries (pour les garçons : la confrérie du Sacré-Cœur 1906, confrérie du Très Saint Enfant 1909, reconnue par la Papauté 
comme Archiconfrérie en 1910, la Sainte Famille à Bethléem) suivent des exercices spirituels et reçoivent généralement les sacrements ${ }^{223}$. La plupart des activités organisées se déroulent en français ; il existe alors peu d'associations catholiques semblables en langue arabe. Au départ ces associations avaient également pour objet de ramener dans le giron du catholicisme les élèves chrétiens non latins :

- 17 religions différentes (la moitié catholiques), presque autant de nationalités, bonne entente (...) discipline et popularité des Frères

- dans les grands collèges, cercle, amicale, conférence de saint Vincent de Paul

- JEC jeunesse étudiante chrétienne

- Je puis comparer ce mouvement à un torrent impétueux qui a tout balayé sur son passage. Les fruits sont merveilleux, les conversions se suivent de plus en plus nombreuses ${ }^{224}$.

${ }^{223}$ Archives de la Maison Généralice et BEC Bulletin des Ecoles Chrétiennes, statuts des confréries et associations.

${ }^{224}$ Extrait, Bulletin CEuvre d'Orient, note du Frère Visiteur Ildefonse, 1930. Ces conversions ne sont inscrites, ni dans les registres du collège, ni dans les archives de la paroisse de Saint Sauveur. S'agit-il d'un effet de rhétorique auprès des Euvres? ou mentionne-t-il le cas syrien, compris alors dans le district de Jérusalem ? 


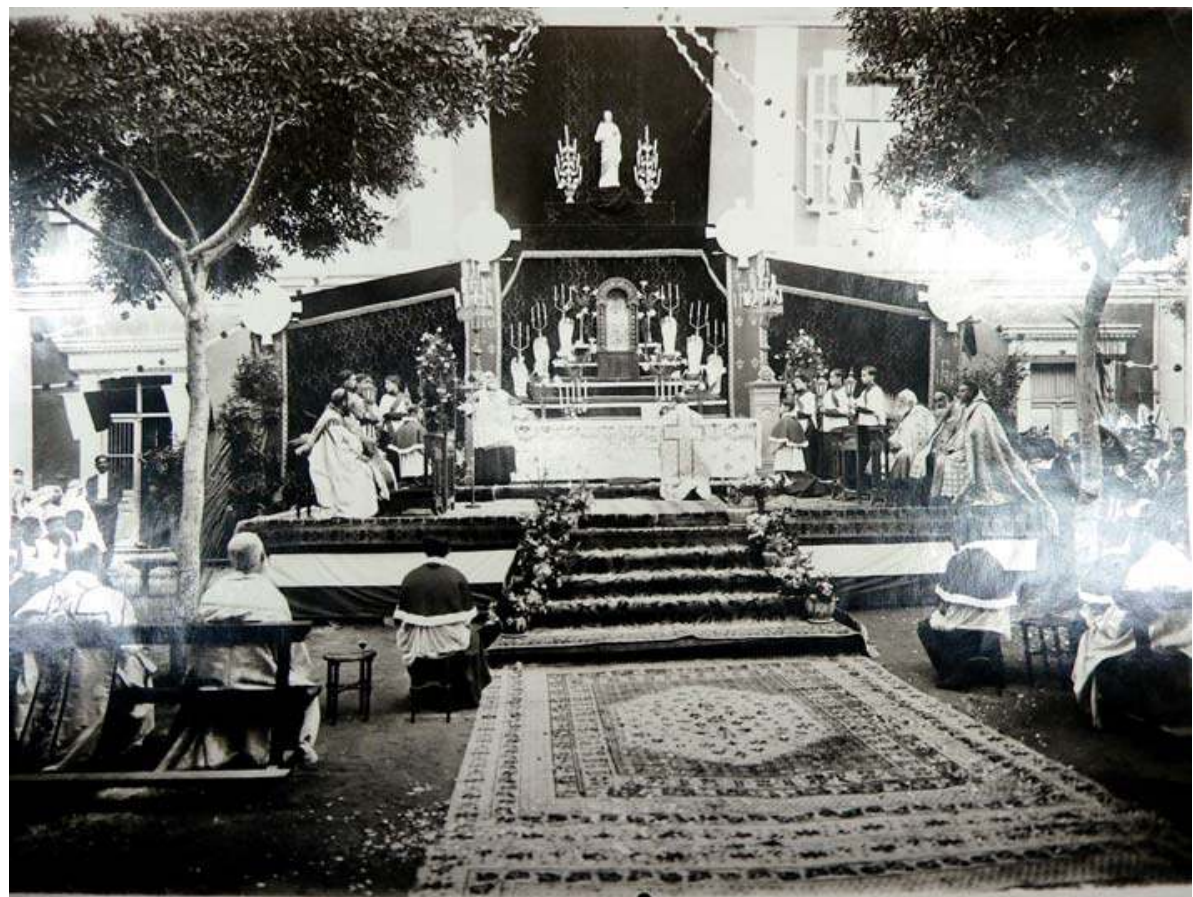

Fête de la Vierge, collège de Jérusalem - Archives du collège -

Les établissements des Sœurs comportent tous les associations de Croisées et la confrérie des Filles de Marie. Au chapitre général de 1934 est exposée l'importance qu'accorde l'Institut à l'Action Catholique. Il s'agit «d'enrôler [les jeunes] dans des groupes où ils auront à agir ${ }^{225}$ ». Pourtant les rapports de visite déplorent parfois dans le cas de la Palestine des actions qui restent trop discrètes, et qui selon le Frère directeur, ne permettent pas d'enrayer l'influence négative des organisations qui ne sont plus liées à la religion ${ }^{226}$.

225 BEDEL, Henri, Etudes lassaliennes 11, 2003, p. 101 ; l'Institut comporte différentes sections: dans les écoles primaires, dans les collèges et écoles techniques, dans les œuvres postscolaires.

${ }^{226}$ Archives de la Maison de Jérusalem, note du Frère Florin, directeur, 1931. Comparativement à la scène culturelle libanaise, surtout celle de Beyrouth, 
En l'espace de 20 ans, la France a changé sa perception des rapports entre langue et religion dans les établissements français catholiques de la Palestine mandataire. Face à la déconsidération de la langue française par les autorités britanniques ${ }^{227}$, le Consulat Général encourage ces ordres à progressivement diffuser non plus seulement une image chrétienne de la France mais encore une image plus universaliste ${ }^{228}$; cette position est bien différente de celles des gouvernements antérieurs aux années 1920.

Le couple « catholique / français » est également perçu de façon différente par les familles des élèves. L'enseignement dispensé au sein de ces établissements va plus loin que l'enseignement de la langue prise comme support de la religion, car c'est l'image d'un modèle culturel à transmettre qui domine. C'est pourquoi le consulat continue à verser à ces établissements des subventions régulières et à les encourager dans leur action. Pourtant, au travers de ces établissements apparaît aussi la réorientation, à partir du milieu des années 1930, de la politique linguistique et culturelle française en Palestine : une politique culturelle qui se s'appuie plus seulement sur ces ordres, représentants de longue date de la France en Palestine. Le Consul général de Jérusalem de 1929 à 1938, le vicomte d'Aumale, est en effet d'avis que la France a peu de perspectives avec le public d'élèves arabes des Frères ${ }^{229}$, et qu'il lui faut alors se tourner également vers le public juif.

Face à cette situation difficile pour leur ordre et pour le Consulat, les Frères privilégient dans l'immédiat la promotion de la langue française, conjointement au renforcement de l'enseignement de

Jérusalem n'accueille pas autant de conférences et événements littéraires. L'instauration d'un Centre de Culture française dans la partie ouest de Jérusalem vise un public davantage juif. MAE Nantes, O 369, documents relatifs à la création et au fonctionnement du CCF, Centre de Culture Française. Les associations dont les Britanniques font la promotion connaissent donc un réel succès, notamment le mouvement des scouts.

${ }^{227}$ Les autorités mandataires ne reconnaissent plus à la langue française le statut de « langue d'examen » dès 1924 ; d'autre part l'anglais devient dès les premières années du Mandat la langue dominante dans la société palestinienne, celle qui désormais permet d'accéder aux fonctions administratives, et l'influence de la langue française diminue.

${ }^{228}$ C'est moins vrai dans le cas des Sœurs de Saint Joseph qui continuent à attirer un public à majorité catholique.

${ }^{229}$ Aumale, J. d'., Voix de l'Orient, souvenirs d'un diplomate, Montréal : Variétés, 1945 ; et correspondances avec le MAE, 1931-1934. 
l'arabe et de l'anglais. Ils se perçoivent en effet comme les défenseurs de la langue davantage que de la religion catholique, sentant la langue (et son enseignement en Palestine) davantage menacée que l'enseignement à vocation catholique pour lequel ils ont été envoyés, ce qui leur est rappelé à de nombreuses occasions par les autorités ecclésiastiques.

Durant toute la période mandataire, les autorités ecclésiastiques demandent explicitement aux établissements d'enseignement de Palestine d'accentuer leurs efforts sur la langue arabe utilisée pour éduquer à la religion catholique, tout en affirmant à plusieurs reprises la volonté de respecter le culte oriental. Ces écoles et collèges doivent également composer avec les aspirations locales pour conserver leurs publics, la demande d' «arabisation» de l'enseignement et l'introduction du Palestine Matriculation ${ }^{230}$ étant plus importantes aux yeux de certaines familles que l'éducation chrétienne en français. L'enseignement de la religion exclusivement en arabe reste un objet de préoccupation à la fin de la période considérée, comme le confirme par exemple le secrétaire des Euvres françaises au secrétaire général des Frères :

Je m'empresse de vous indiquer que nous sommes intervenus à Rome au sujet de la langue dans laquelle est enseigné le catéchisme en Palestine [...] je puis vous faire savoir confidentiellement que son Eminence le cardinal secrétaire de la Congrégation de l'Eglise Orientale estime que le catéchisme devrait être enseigné en arabe dans les basses classes et en français dans les classes moyennes et supérieures $^{231}$.

Les consulats français de Jérusalem et auprès du Saint-Siège servent encore parfois d'intermédiaires entre les établissements, leur hiérarchie et les autorités romaines :

1- l'enseignement du catéchisme a pour but de mettre à la portée des élèves et si possible de leurs familles, les vérités de la foi ; or il est incontestablement plus facile d'atteindre ce but si l'enseignement est fait dans leur propre langue.

\footnotetext{
${ }^{230}$ Examen officiel de fin d'enseignement secondaire de Londres, mis en place dès le début du Mandat.

${ }^{231}$ MAE œuvres françaises, $n^{\circ} 362,13$ décembre 1941.
} 
$[\ldots]$.

4- la réfutation des erreurs des schismatiques, des protestants, des musulmans, réfutation qui présente un intérêt particulier pour la formation de la jeunesse catholique de Palestine, se trouve dans la partie du catéchisme diocèse qui est destinée aux classes moyennes et supérieures.

5- si l'on faisait une exception aux Frères, on ne saurait la refuser aux autres Congrégations, de différentes nationalités, qui se consacrent à l'enseignement dans le diocèse ${ }^{232}$.

Mais à la fin de la période, le caractère professionnel de l'enseignement de ces établissements est confirmé, ils semblent devoir en priorité éduquer à la langue et à la culture française avant d'être un instrument au service de la religion catholique.

\section{BIBLIOGRAPHIE}

AUDISIO, Gabriel, dir., Religion et identité, Aix en Provence : Publications de l'université de Provence, 1998.

BOWMAN, H.E., Middle East Window, Londres: Longman, 1942.

BURROWS, M., "Mission civilisatrice, French Cultural Policy in the Middle East, 1860-1914 », The Historical Journal, 29, I, 1986, p. 109-135.

CABANEL, Patrick, Une France en Méditerranée, écoles, langues et cultures françaises $X I X^{e}, X X^{e}$ siècles, Grâne : Créaphis, 2006.

AUMALE, J. d', Voix de l'Orient, souvenirs d'un diplomate, Montréal : Variétés, 1945.

KHALIDI, Rashid, Palestinian identity, the construction of a modern national identity, New York: Columbia University Press, 1997.

LAURENS, Henri, Orientales III, parcours et situations, Paris : CNRS éditions, 2004.

${ }^{232}$ Secrétaire du Vatican, 10 août 1942, en réponse à la lettre de décembre 1941 de l'ambassade de France près du Saint-Siège (demande d'explications). 
NICAULT, Catherine, « La fin du protectorat catholique de la France à Jérusalem, 1918-1924 », Bulletin du CRFJ, 1999.

PIERRACCINI, Paolo, «Un Universita cattolica a Gerusalemme? L'Opera cardinal Ferrari e il collegio francescano Di Terra Santa », Antonianum LXXXI, 1, Rome: Pontificiae Universitatis Antonianum, 2006.

TIBAWI, Abdul Latif, Arab Education in Mandatory Palestine : a story of three decades of British administration, Londres: Luzac, 1956.

TRIMBUR, Dominique, "L'ambition culturelle de la France en Palestine dans l'entre deux guerres", in Entre rayonnement et réciprocité. Contributions à l'histoire de la diplomatie culturelle, Paris: Publications de la Sorbonne, Série Internationale Paris I Panthéon Sorbonne, 2002, p. 41-72.

Prospectus du collège de Jérusalem (non daté mais d'après les descriptifs indirects des enseignements et les entretiens oraux, très certainement d'avant 1945) - Archives du collège - 


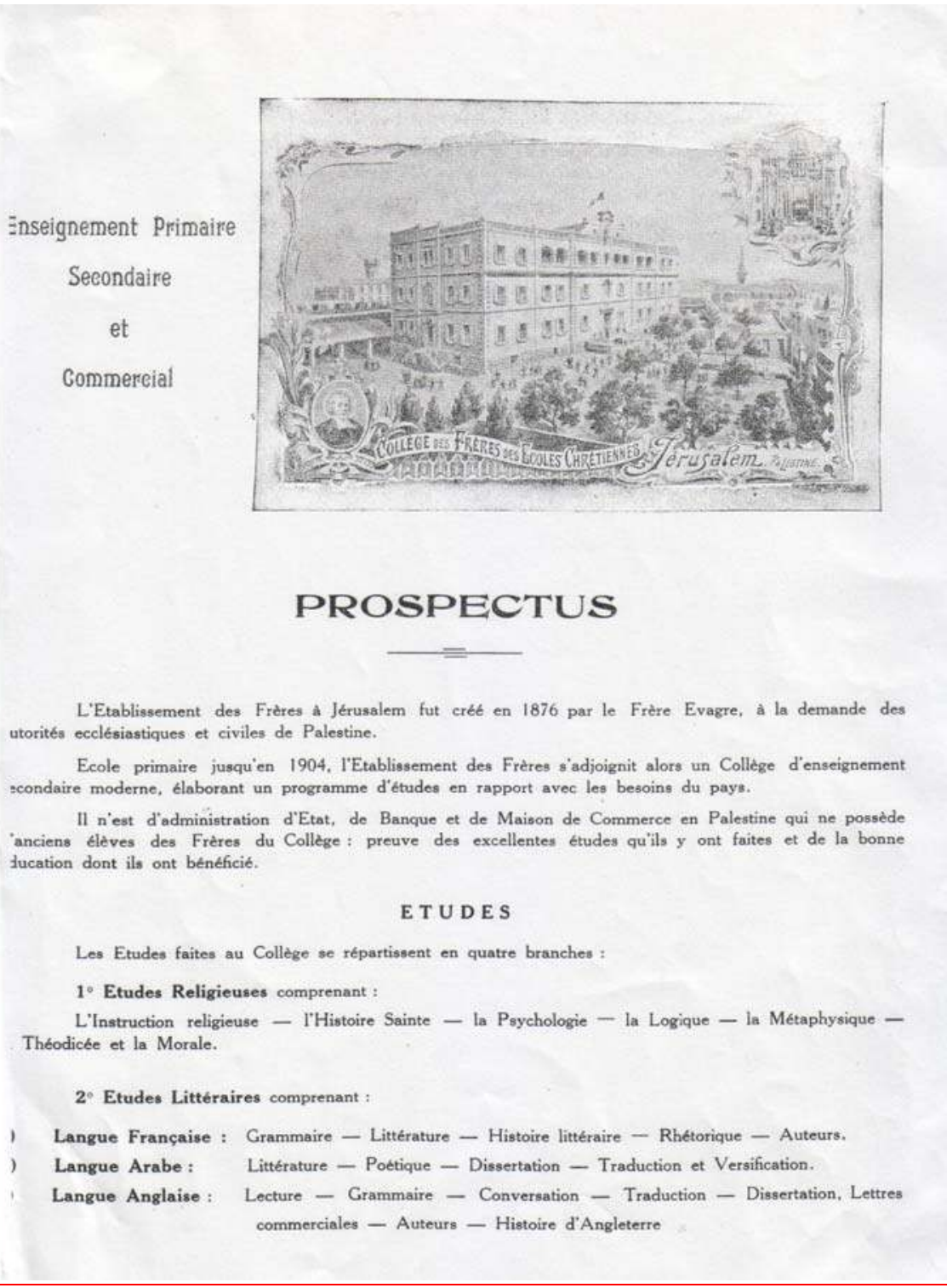

